\title{
Stochastic Source Seeking by Mobile Robots
}

\author{
Shun-ichi Azuma, Member, IEEE, Mahmut Selman Sakar, Member, IEEE, and George J. Pappas, Fellow, IEEE
}

\begin{abstract}
We consider the problem of designing controllers to steer mobile robots to the source (the minimizer) of a signal field. In addition to the mobility constraints, e.g., posed by the nonholonomic dynamics, we assume that the field is completely unknown to the robot and the robot has no knowledge of its own position. Furthermore, the unknown field is randomly switching. In the case where the information of the field (e.g., the gradient) is completely known, standard motion planning techniques for mobile robots would converge to the known source. In the absence of mobility constraints, convergence to the minimum of unknown fields can be pursued using the framework of numerical optimization. By considering these facts, this paper exploits an idea of the stochastic approximation for solving the problem mentioned in the beginning and proposes a source seeking controller which sequentially generates the next waypoints such that the resulting discrete trajectory converges to the unknown source and which steers the robot along the waypoints, under the assumption that the robot can move to any point in the body fixed coordinate frame. To this end, we develop a rotation-invariant and forward-sided version of the simultaneous-perturbation stochastic approximation algorithm as a method to generate the next waypoints. Based on this algorithm, we design source seeking controllers. Furthermore, it is proven that the robot converges to a small set including the source in a probabilistic sense if the signal field switches periodically and sufficiently fast. The proposed controllers are demonstrated by numerical simulations.
\end{abstract}

Index Terms-Mobile robots, nonholonomic systems, simultaneous-perturbation stochastic approximation, source seeking.

\section{INTRODUCTION}

$\mathbf{S}$ OURCE seeking is a mixed problem of search and navigation as shown in Fig. 1: when a mobile robot is placed in an environment where an unknown signal field, i.e., an unknown spatial profile of the signal, is introduced, find a controller to steer the robot to the source (the unknown minimizer) without using the position information. The field is given by a scalar-valued function, denoted by $f(x)$ in Fig. 1, which could express the spatial distribution of magnetic force, heat, or chemical concentration. The robot is navigated by only using the measurements of the signal at the positions.

Manuscript received March 25, 2010; revised July 24, 2010; accepted December 21, 2011. Date of publication February 03, 2012; date of current version August 24, 2012. This work was supported by the ONR-MURI award N00014-08-1-0696 and The Kyoto University Foundation. Recommended by Associate Editor M. Egerstedt.

S.-i. Azuma is with Graduate School of Informatics, Kyoto University, Kyoto 611-0011, Japan (e-mail: sazuma@i.kyoto-u.ac.jp).

M. S. Sakar is with Mechanical Engineering Department, Massachusetts Institute of Technology, Cambridge, MA 01239 USA (e-mail: selman@mit.edu).

G. J. Pappas is with Department of Electrical and Systems Engineering, University of Pennsylvania, Philadelphia, PA 19104 USA (e-mail: pappasg@seas. upenn.edu).

Color versions of one or more of the figures in this paper are available online at http://ieeexplore.ieee.org.

Digital Object Identifier 10.1109/TAC.2012.2186927

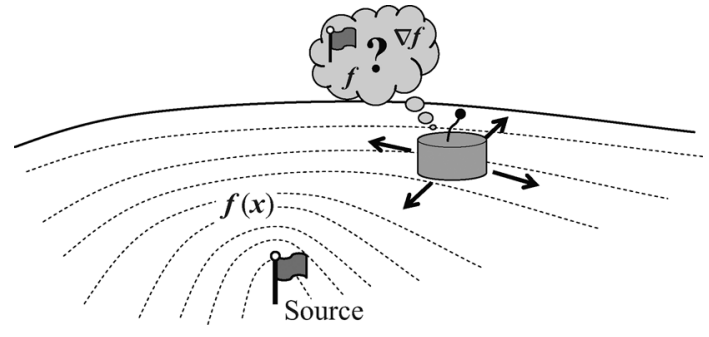

Fig. 1. Source seeking problem.

This topic will have great potential for a wide range of future applications, including wireless communication, medical science, security engineering, and natural resource development. For example, the robotic suspect search is considered for the concentration field of a smell substance, and the landmine search is done for a magnetic field (where the strongest signal point is regarded as the minimizer of a function quantifying the weakness field). Another possible application is the robotic pinpoint dose for avoiding side effects, where, instead of diffusing a medicine in the body, the medicine is directly sent to invisible tumor cells by a micro robot. In this case, a protein concentration field is used (related techniques are found in, e.g., [1], [2]).

Currently, there are three main approaches to the problem. In [3]-[5], mathematical programming-based methods have been provided, where a gradient type controller and a hybrid controller have been given. An approach based on random walk has been proposed in [6]. There, it has been shown that the probability distribution on the robot position converges to a desired function. The extremum seeking technique [7], originally developed for adaptive control, has been applied in [8]-[14]. Apart from these approaches, related problems have been discussed in [15]-[20], where, unlike the situation considered here, it is assumed that the position information is available for the navigation, or the problems do not include any control issue of mobile robots.

Here, we are interested in the stochastic source seeking, which involves a randomly switching field. This is motivated by the following fact. Although switching fields appear in many applications, such a situation has never been handled so far, except for a mathematically similar case with noisy signal fields [13]. An example with a switching field is the base station placement for wireless communication, which is to find the best location in terms of the terminal density. In this case, the signal field corresponds to the radio field made by a number of terminal units, which randomly switches depending on their usage, and the source is, for example, the strongest signal point in an expectation sense. This example is closely related to sensor networks, which is an actively studied topic in recent years, where the terminal units correspond to sensor 
nodes, and each node switches its own state between the active mode and the sleep mode for energy saving.

This paper thus establishes a framework of stochastic source seeking by mobile robots. Our approach is to find a controller which sequentially generates the next waypoints such that the resulting discrete trajectory converges to the unknown source and which steers the robot along the waypoints, under the assumption that the robot can move to any point in the body fixed coordinate frame. Here, the idea of a stochastic approximation technique, called the simultaneous-perturbation stochastic approximation (SPSA) [21], is utilized to obtain the waypoints. The contributions of this paper are summarized as follows.

First, we extend the SPSA algorithm to an appropriate form for the source seeking by mobile robots. Since the original algorithm generates the waypoints based on the world coordinate frame, it is impossible to drive the robot along the waypoints without a position sensor. In contrast, the new algorithm provides the waypoints in a time-varying coordinate frame, which fits the sensor-free navigation. Based on this, we present source seeking controllers composed of point-to-point controllers. It is then proven that the controllers drive the robot to a small set including the source with probability 1 if the field switches periodically and faster than the measurement period of the signal.

Second, simple source seeking controllers are presented for two- and three-dimensional nonholonomic robots by exploiting a special structure. This shows that the stochastic source seeking is achieved by repeating two actions: a random turn and a forward/backward move.

It should be stressed that this paper does not just apply an existing optimization method to the source seeking problem. In fact, it is not true that any optimization method can be employed, because the optimization method for our problem must have the following properties: 1) the method uses the measurements of the function $f$, instead of the explicit form of $f$ and $\nabla f$ (see Fig. 1); 2) the number of measurements has to be small for efficiency; 3) the method can handle stochastic switching fields; and 4) the waypoints given by the method can be followed by the robot with no position sensor. In this paper, by focusing on these points, the SPSA algorithm [21] is picked from a number of optimization techniques, and is extended to a suitable version for the source seeking. It is also noted that, as a first step to the source seeking with stochastically switching fields, we mainly consider a somewhat limited case, where the fields switch periodically and sufficiently fast. This may limit possible applications, but the paper will be a basis in developing stochastic source seeking methods for more general cases.

This paper is organized as follows. In Section II, the stochastic source seeking problem is formulated and the idea for the solution is outlined. Next, a generalized version of the SPSA algorithm is provided in Section III, and our source seeking controllers are presented in Section IV. Section V concludes this paper.

This paper is based on our earlier preliminary version [22], and contains full explanations and proofs omitted there.

\section{Notation}

Let $\mathbf{R}, \mathbf{R}_{+}, \mathbf{R}_{0+}$, and $\mathbf{N}$ be the real number field, the set of positive real numbers, the set of nonnegative real numbers, and the set of nonnegative integers, respectively. We denote by

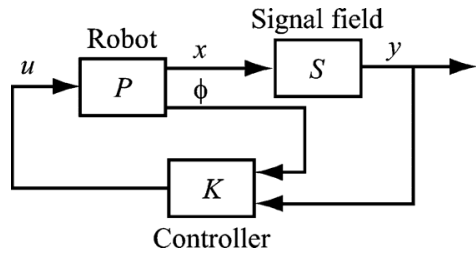

Fig. 2. Control system for source seeking.

$0_{n \times m}$ and $I_{n}$ (or for simplicity of notation, 0 and $I$ ) the $n \times m$ zero matrix and the $n \times n$ identity matrix. For the vector $x \in \mathbf{R}^{n}$, we use $\|x\|$ and $\operatorname{sign}(x)$ to express the Euclidian norm and the signum vector. If the vector $x$ is composed of nonzero elements, let $x^{(-1)}$ be the vector composed of the elementwise inverse, i.e., $x^{(-1)}=\left[\begin{array}{llll}x_{1}^{-1} x_{2}^{-1} & \cdots & x_{n}^{-1}\end{array}\right]^{\top} \in \mathbf{R}^{n}$ where $x_{i}$ is the $i$ th element of $x$. The vectorization of the matrix $M$ is expressed by $\operatorname{vec}(M)$. For the number $\theta \in \mathbf{R}, R_{2}(\theta)$ is the two-dimensional rotation matrix defined as

$$
R_{2}(\theta):=\left[\begin{array}{rr}
\cos \theta & -\sin \theta \\
\sin \theta & \cos \theta
\end{array}\right] .
$$

Furthermore, $R_{3}(\psi)$ expresses the three-dimensional rotation matrix with the yaw, pitch, and roll specified by the vector $\psi:=$ $\left[\begin{array}{lll}\psi_{1} & \psi_{2} & \psi_{3}\end{array}\right]^{\top} \in \mathbf{R}^{3}$ in radians. Note that the matrices are orthogonal, e.g., $R_{2}(\theta) R_{2}^{\top}(\theta)=I_{2}$, which plays an important role in this paper. For the number $a \in \mathbf{R}$, let $\lfloor a\rfloor$ be the maximum integer less than or equal to $a$. The scalar/vector/function sequence $\left\{x_{l}, x_{l+1}, \ldots, x_{m}\right\}$ is denoted by $\left\{x_{i}\right\}_{i=l}^{m}$ and, for simplicity, it is denoted by $\left\{x_{i}\right\}$ if $l=0$ and $m=\infty$. The gradient of the scalar-valued function $f: \mathbf{R}^{n} \rightarrow \mathbf{R}$ is denoted by $\nabla f(x)$, i.e.,

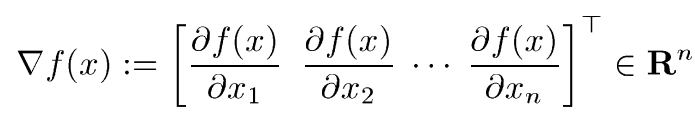

where $x_{i}$ is the $i$ th element of the vector $x \in \mathbf{R}^{n}$. Finally, $\mathbf{S}_{1} \oplus$ $\mathbf{S}_{2}$ represents the Minkowski sum of the sets $\mathbf{S}_{1}$ and $\mathbf{S}_{2}$.

\section{Stochastic Source Seeking Problem}

\section{A. Problem Formulation}

Consider the feedback system in Fig. 2, composed of the mobile robot $P$, the signal field $S$, and the controller $K$.

The robot $P$ is given by

$$
P:\left[\begin{array}{c}
\dot{x}(t) \\
\dot{\theta}(t) \\
\dot{\phi}(t)
\end{array}\right]=G(x(t), \theta(t), \phi(t)) u(t)
$$

where $x(t) \in \mathbf{R}^{n_{1}}$ and $\theta(t) \in \mathbf{R}^{n_{2}}$ are the translational and orientational positions in the world coordinate frame, $\phi(t) \in \mathbf{R}^{n_{3}}$ is the other state variable defined relative to the absolute position $(x(t), \theta(t))$ and is called the internal posture, $u(t) \in \mathbf{R}^{m}$ is the control input, and $G: \mathbf{R}^{n_{1}} \times \mathbf{R}^{n_{2}} \times \mathbf{R}^{n_{3}} \rightarrow \mathbf{R}^{\left(n_{1}+n_{2}+n_{3}\right) \times m}$ is a nonlinear function describing the dynamics. We assume that $P$ is in a two- or three-dimensional space, i.e., $\left(n_{1}, n_{2}\right) \in$ $\{(2,1),(3,3)\}$. It is well-known that many drift-free mobile robots can be expressed by (1) [23]. An example of $P$ is the 


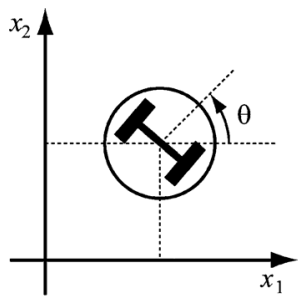

(a)

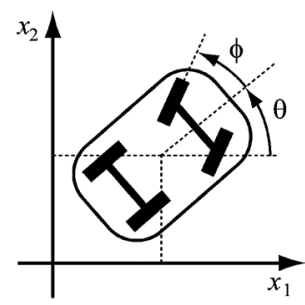

(b)
Fig. 3. Examples of mobile robots. (a) Unicycle. (b) Four-wheeled vehicle.

Kinematic model of the nonholonomic unicycle in Fig. 3(a), which is described by

$$
\left[\begin{array}{c}
\dot{x}_{1}(t) \\
\dot{x}_{2}(t) \\
\dot{\theta}(t)
\end{array}\right]=\left[\begin{array}{cc}
\cos \theta(t) & 0 \\
\sin \theta(t) & 0 \\
0 & 1
\end{array}\right] u(t)
$$

where $x_{1}(t) \in \mathbf{R}, x_{2}(t) \in \mathbf{R}, \theta(t) \in \mathbf{R}$, and $u(t) \in \mathbf{R}^{2}$. The state variable $\phi$ for the internal posture is not required for the unicycle but will be used for more complicated robots such as the four-wheeled vehicle in Fig. 3(b) where the steering angle is expressed by $\phi$.

The signal field $S$ is a transducer from the information on $x$ to a scalar signal, which is of the randomly switching form

$$
S: y(t)=f_{\sigma(t)}(x(t))
$$

where $y(t) \in \mathbf{R}$ expresses the signal strength and $f_{\sigma}: \mathbf{R}^{n_{1}} \rightarrow$ $\mathbf{R}$ are thrice differentiable functions with respect to $x$. Furthermore, $\sigma(t) \in\{1,2, \ldots, N\}$ is the piecewise constant random signal given as $\sigma(t)=\sigma_{i}$ on the time interval $[i \eta,(i+1) \eta)$ where $i \in \mathbf{N}, \sigma_{i}$ are the independent and identically distributed (i.i.d.) random variables from the probability distribution $g$ : $\{1,2, \ldots, N\} \rightarrow[0,1]$, and $\eta \in \mathbf{R}_{+}$is the switching time period. An example of $S$ is shown in Section IV-C. We denote by $E\left[f_{\sigma}(x) \mid x\right]$ the conditional expected value $\sum_{\sigma=1}^{N} g(\sigma) f_{\sigma}(x)$, i.e., $E\left[f_{\sigma}(x) \mid x\right]=\sum_{\sigma=1}^{N} g(\sigma) f_{\sigma}(x)$, and we call the local minimizer(s) of $E\left[f_{\sigma}(x) \mid x\right]$ the source(s), where note that $\sigma$ corresponds to just a random variable drawn from the probability distribution $g$. For example, if $n_{1}:=2, N:=2, f_{1}(x):=x^{\top} x$, $f_{2}(x):=\left(x-\left[\begin{array}{ll}0 & 1\end{array}\right]^{\top}\right)^{\top}\left(x-\left[\begin{array}{ll}0 & 1\end{array}\right]^{\top}\right), g(\sigma)=0.5$ for $\sigma \in\{1,2\}$, and $g(\sigma)=0$ for $\sigma \notin\{1,2\}$, then

$$
\begin{aligned}
E\left[f_{\sigma}(x) \mid x\right] & =0.5 x^{\top} x+0.5\left(x-\left[\begin{array}{ll}
0 & 1
\end{array}\right]^{\top}\right)^{\top}\left(x-\left[\begin{array}{ll}
0 & 1
\end{array}\right]^{\top}\right) \\
& =x^{\top} x-\left[\begin{array}{ll}
0 & 1
\end{array}\right] x+0.5
\end{aligned}
$$

and the source is $x=\left[\begin{array}{ll}0 & 0.5\end{array}\right]^{\top}$.

The controller $K$ is a (causal) dynamical system which could be a continuous-time system or a discrete-time system with a sampler and a holder. The inputs are $\phi$ and $y$, which means that the information on the internal posture $\phi$ is available by some internal sensors of $P$ (e.g., potentiometers) but the absolute position $(x, \theta)$ is not.

Then we consider the following problem.

Problem 1 (Stochastic Source Seeking): For the feedback system in Fig. 2, suppose that $P, S$, and a positive number $r \in \mathbf{R}_{+}$are given, but assume that $S$ is unknown (i.e., the functions $f_{\sigma}(\sigma=1,2, \ldots, N)$ and the probability distribution $g$ are unknown). Then find a controller $K$ seeking a source, i.e., a $K$ such that there exists an initial state set $\mathbf{X}_{0} \in \mathbf{R}^{n_{1}}$ satisfying

1) $\mathbf{X}_{0}$ is a nonzero-measure set including a source in its interior;

2) for every $\left(x_{0}, t\right) \in \mathbf{X}_{0} \times \mathbf{R}_{0+},\|x(t)\|<\infty$ w.p.1 under $x(0)=x_{0}$;

3) for each $x(0)=x_{0} \in \mathbf{X}_{0}$, there exists a time instant $t_{s} \in \mathbf{R}_{+}$satisfying

$$
\left\|x(t)-x^{*}\right\| \leq r
$$

for every $t \in\left[t_{s}, \infty\right)$ w.p. 1 where $x^{*} \in \mathbf{X}_{0}$ is the source.

Several remarks on this problem are given.

First, 3 ) is the condition on the convergence not to a source but to a closed ball including a source. This is fairly standard for source seeking problems, since the trajectories of the robot $P$ are often restricted by the mobility constraints (such as nonzero velocity constraints and nonholonomic constraints) and the convergence to a single point is often impossible. Note here that, if the given $r$ is smaller than a value depending upon the mobility constraint, then it is concluded that the problem is infeasible, i.e., there is no solution to this problem.

Next, we have no information on $S$ except for a few assumptions, which poses two challenging issues in this problem. First, even if we focus on only the static optimization problem

$$
\min _{x \in \mathbf{R}^{n_{1}}} E\left[f_{\sigma}(x) \mid x\right]
$$

typical methods, using the explicit form of $E\left[f_{\sigma}(x) \mid x\right]$ or its gradient, cannot be employed (because we do not have the expression of $\left.E\left[f_{\sigma}(x) \mid x\right]\right)$. Namely, our attention has to be restricted to methods only using the measurements of $f_{\sigma(t)}(x(t))$. Second, in the feedback system in Fig. 2, it is impossible to estimate the absolute position of $P$ through the measurements of $y$. Thus, $K$ has to generate the control input without using the position information, which prevents us from applying position control methods based on the world coordinate frame.

Finally, it is assumed in the problem that the robot $P$ has a single sensor to measure the signal strength (i.e., $y(t) \in \mathbf{R}$ ) of the signal field $S$. On the other hand, if $P$ has multiple sensors appropriately embedded, the gradient information of $S$ can be directly obtained and utilized for source seeking. Nevertheless, this paper does not deal with such a situation, because we are interested in the source seeking with the minimum number of sensors.

\section{B. Solution Idea and Preparation}

The idea to solve Problem 1 is outlined as follows. As easily imagined, Problem 1 raises two issues: the exploration of the solution to the static problem (4), and the control of the robot. As a solution to the former, we first present a set of stochastic discrete trajectories (almost surely) converging to a solution to (4). Next, we pick an appropriate stochastic trajectory from the set and give a controller $K$ to steer the robot along the trajectory, which solves the latter. These will be respectively detailed in the next two sections. 
In considering the approach, some symbols are prepared at this point. For the robot $P$, we often use the body fixed coordinate frame. The frame at time $\tau$ is denoted by $\Sigma(\tau)$, i.e.,

$$
\Sigma(\tau):\left[\begin{array}{c}
z(t) \\
\psi(t) \\
\varphi(t)
\end{array}\right]=\left[\begin{array}{c}
R_{n_{1}}(-\theta(\tau))(x(t)-x(\tau)) \\
\theta(t)-\theta(\tau) \\
\phi(t)
\end{array}\right]
$$

where $t$ expresses a future time after $\tau,(z(t), \psi(t), \varphi(t)) \in$ $\mathbf{R}^{n_{1}} \times \mathbf{R}^{n_{2}} \times \mathbf{R}^{n_{3}}$ are the new coordinates, and $R_{n_{1}}(-\theta(\tau))$ is the $n_{1}$-dimensional rotation matrix defined in Section I. If

(P1) for each $(\Delta z, \Delta \psi) \in \mathbf{R}^{n_{1}} \times \mathbf{R}^{n_{2}}$, there exists a control input $u$ such that:

1) $\|z(t)\| \leq r_{1}\|\Delta z\|+r_{2}\|\Delta \psi\|+r_{3}$ for every $t \in$ $[\tau, \tau+\Delta \tau]$

2) $z(\tau+\Delta \tau)=\Delta z$ and $\psi(\tau+\Delta \tau)=\Delta \psi$

under the conditions $z(\tau)=0$ and $\psi(\tau)=0$

holds for some $\Delta \tau \in \mathbf{R}_{+}$and $\left(r_{1}, r_{2}, r_{3}\right) \in \mathbf{R}_{0+} \times \mathbf{R}_{0+} \times \mathbf{R}_{0+}$, we denote by

$$
v\left(t, \Delta \tau, 0 \rightarrow\left[\begin{array}{c}
\Delta z \\
\Delta \psi
\end{array}\right], r_{1}, r_{2}, r_{3}\right)
$$

a control input (function) for $t \in[\tau, \tau+\Delta \tau]$ to steer the robot $P$ as stated in (P1). Note that (P1) corresponds to a kind of controllability assumption, which guarantees the existence of a point-to-point controller to steer $P$ from the current position to $\left[(\Delta z)^{\top}(\Delta \psi)^{\top}\right]^{\top}$ keeping the boundedness of $z(t)$ on the time interval $[\tau, \tau+\Delta \tau]$. Note also that applying $v$ to $P$ results in

$$
\begin{aligned}
{\left[\begin{array}{l}
x(\tau+\Delta \tau) \\
\theta(\tau+\Delta \tau)
\end{array}\right] } & =\left[\begin{array}{l}
x(\tau) \\
\theta(\tau)
\end{array}\right]+\left[\begin{array}{c}
R_{n_{1}}(\theta(\tau)) \Delta z \\
\Delta \psi
\end{array}\right] \\
\|x(t)-x(\tau)\| \leq & \leq r_{1}\|\Delta z\|+r_{2}\|\Delta \psi\|+r_{3}(\forall t \in[\tau, \tau+\Delta \tau])
\end{aligned}
$$

in the world coordinate frame. A typical method to obtain the input $v$ is to utilize the so-called Lie bracket motion based on periodic inputs and the accessibility distribution ${ }^{1}$ of $P$ (see, e.g., [24]).

\section{STOChASTIC Discrete TRAJECTORIES FOR Stochastic SOURCE SeEking}

To obtain stochastic discrete trajectories for the source seeking, we employ the idea of a stochastic approximation technique, called the simultaneous-perturbation stochastic approximation (SPSA) [21]. Since the original SPSA algorithm is not applicable to our source seeking problem as stated in Section I (which will be detailed in Remark 2), we extend the original algorithm to a more suitable version.

\section{A. Generalized Simultaneous-Perturbation Stochastic Approximation}

A general form of the stochastic approximation algorithm is given by

$$
x_{k+1}=x_{k}-a_{k} d\left(x_{k}, c_{k}, \xi_{k}, \delta_{k}\right)
$$

where $x_{k} \in \mathbf{R}^{n}$ is the state, $a_{k} \in \mathbf{R}_{+}$and $c_{k} \in \mathbf{R}_{+}^{l}$ are the gains, $\xi_{k} \in \mathbf{R}^{p}$ is the random variable introduced for solving a

${ }^{1}$ The linear combination of the all Lie brackets for the column vectors of $G(x, \theta, \phi)$. problem, $\delta_{k} \in \mathbf{R}^{q}$ is the variable expressing noise and uncertainty, and $d: \mathbf{R}^{n} \times \mathbf{R}_{+}^{l} \times \mathbf{R}^{p} \times \mathbf{R}^{q} \rightarrow \mathbf{R}^{n}$ is the search direction. This algorithm is closely related to the steepest descent, which corresponds to the case $d\left(x_{k}, c_{k}, \xi_{k}, \delta_{k}\right)=\nabla f\left(x_{k}\right)$ for the function $f: \mathbf{R}^{n} \rightarrow \mathbf{R}$ to be minimized.

For the algorithm in (9), we propose the search direction

$$
\begin{aligned}
& d\left(x_{k}, c_{k}, \xi_{k}, \delta_{k}\right):= \\
& T_{k} \frac{\left(f\left(x_{k}+c_{k 1} T_{k} \xi_{k}\right)+\varepsilon_{k+}\right)-\left(f\left(x_{k}-c_{k 2} T_{k} \xi_{k}\right)+\varepsilon_{k-}\right)}{\left(c_{k 1}+c_{k 2}\right)} \xi_{k}^{(-1)}
\end{aligned}
$$

for $c_{k}:=\left[\begin{array}{ll}c_{k 1} & c_{k 2}\end{array}\right]^{\top}$ and $\delta_{k}:=\left[\begin{array}{lll}\varepsilon_{k+} & \varepsilon_{k-} & \operatorname{vec}\left(T_{k}\right)\end{array}\right]^{\top}$, where $f: \mathbf{R}^{n} \rightarrow \mathbf{R}$ is the function to be minimized, $c_{k 1}, c_{k 2} \in$ $\{0\} \cup \mathbf{R}_{+}$are the gains, $\xi_{k} \in \mathbf{R}^{n}(p=n)$ is the random variable, $\xi_{k}^{(-1)}$ is the elementwise inverse of $\xi_{k}$ as defined in Section I, $\varepsilon_{k+}, \varepsilon_{k-} \in \mathbf{R}$ are the random noise, and $T_{k} \in \mathbf{R}^{n \times n}$ is the uncertain time-varying matrix (which will be treated as an uncertain time-varying "rotation" matrix in Section IV). To appropriately define (10), it is assumed that $\left\|c_{k}\right\|>0$, i.e., $c_{k 1}>0$ or $c_{k 2}>0$. We also assume that $T_{k} \in \mathbf{T}$ for the uncertain matrix set $\mathbf{T} \subseteq \mathbf{R}^{n \times n}$ and assume that the random variable $\xi_{k}$ follows the probability distribution $\Xi_{k}: \mathbf{R}^{n} \rightarrow[0,1]$. In the algorithm given by (9) and (10), the problem parameters (given in advance) are the function $f$, the probability distributions of $\left\{\varepsilon_{k+}\right\},\left\{\varepsilon_{k-}\right\}$, and the uncertain matrix set $\mathbf{T}$, while the design parameters of the algorithm are the gain sequences $\left\{a_{k}\right\}$, $\left\{c_{k 1}\right\},\left\{c_{k 2}\right\}$ and the probability distribution sequence $\left\{\Xi_{k}\right\}$. We call the algorithm given by (9) and (10) the generalized simultaneous-perturbation stochastic approximation algorithm or simply the G-SPSA algorithm.

For the algorithm, we discuss here the robust stability, i.e., the convergence for every possible uncertain matrices. In the following part of this section, the conditions and propositions on the solution $x_{k}$ of the G-SPSA algorithm are assumed to be those satisfied for every $\left\{T_{k}\right\} \in \prod_{k=1}^{\infty} \mathbf{T}$, though the universal quantification for the uncertain matrix is omitted for simplicity of notation.

Under several conditions, the G-SPSA algorithm solves the static optimization problem

$$
\min _{x \in \mathbf{R}^{n}} f(x)
$$

by using noisy measurements of $f(x)$. This is formalized in Propositions 1 and 2.

Proposition 1: Consider the search direction $d\left(x_{k}, c_{k}, \xi_{k}, \delta_{k}\right)$ in (10) and let $\xi_{k i} \in \mathbf{R}$ be the $i$ th element of the random vector $\xi_{k}$. If

- the conditions on the problem parameters:

(A1) $f$ is thrice differentiable;

(A2) $E\left[\varepsilon_{k+}-\varepsilon_{k-} \mid\left\{x_{0}, x_{1}, \ldots, x_{k}\right\}, \xi_{k}\right]=0$ w.p. 1 for all $k \in \mathbf{N}$;

(A3) each element of $\mathbf{T}$ is an orthogonal matrix;

- the conditions on the design parameters:

(B1) a) for each $k \in \mathbf{N}, \xi_{k}$ is integrable, the probability distribution $\Xi_{k}$ is symmetric about zero (i.e., $E\left[\xi_{k}\right]=$ $0)$, and there exists a $\beta_{1} \in \mathbf{R}_{+}$such that $\left|\xi_{k i}\right| \leq \beta_{1}$ and 
$\left|\xi_{k i}^{-1}\right| \leq \beta_{1}$ w.p.1 for all $i \in\{1,2, \ldots, n\}$; b) for each $k \in \mathbf{N}, \xi_{k i}(i=1,2, \ldots, n)$ are mutually independent hold, then

$$
E\left[d\left(x_{k}, c_{k}, \xi_{k}, \delta_{k}\right) \mid x_{k}\right]=\nabla f\left(x_{k}\right)+O\left(\left\|c_{k}\right\|^{2}\right)\left(\text { as } c_{k} \rightarrow 0\right)
$$

for every $T_{k} \in \mathbf{T}$, where the left hand side expresses the expected value with respect to $\xi_{k}, \varepsilon_{k+}$, and $\varepsilon_{k-}$.

Proof: See Appendix I.

Proposition 1 implies that, under several assumptions, the expected value of $d\left(x_{k}, c_{k}, \xi_{k}, \delta_{k}\right)$ is nearly equal to the gradient of $f\left(x_{k}\right)$. So the algorithm given by (9) and (10) can be regarded as an approximation of the so-called fixed-point iteration for finding a root of $\nabla f(x)=0$.

Next, the following result is obtained from Proposition 1.

Proposition 2: For the G-SPSA algorithm given by (9) and (10), suppose that a set $\hat{\mathbf{T}} \subset \mathbf{R}^{n \times n}$ satisfying $\mathbf{T} \subseteq \hat{\mathbf{T}}$ is given, and assume that there exists a root $x^{*} \in \mathbf{R}^{n}$ of the equation $\nabla f(x)=0$. Let $\hat{x}_{k}$ denote the state of the modified G-SPSA algorithm so that $\mathbf{T}$ is replaced with $\hat{\mathbf{T}}$. If:

- the conditions on the problem parameters: (A1)-(A3) and (A4) $x^{*}$ is an asymptotically stable equilibrium of $\dot{x}(t)=-\nabla f(x(t))$ (in the Lyapunov sense);

(A5) there exists an $\alpha_{5} \in \mathbf{R}_{+}$such that $E\left[\varepsilon_{k+}^{2}\right] \leq \alpha_{5}$ and $E\left[\varepsilon_{k-}^{2}\right] \leq \alpha_{5}$ for all $k \in \mathbf{N}$;

(A6) $\mathbf{T}$ is a finite set;

- the conditions on the design parameters: (B1) and

(B2) $\lim _{k \rightarrow \infty} a_{k}=0, \sum_{k=0}^{\infty} a_{k}=\infty, \lim _{k \rightarrow \infty} c_{k}=$ 0 , and $\sum_{k=0}^{\infty} a_{k}^{2} /\left\|c_{k}\right\|^{2}<\infty$;

(B3) the random vectors $\xi_{k}(k=0,1, \ldots)$ are mutually independent;

(B4) there exists a $\beta_{4} \in \mathbf{R}_{+}$such that $E\left[\xi_{k i}^{-2}\right] \leq \beta_{4}$ for all $(k, i) \in \mathbf{N} \times\{0,1, \ldots, n\}$;

- the conditions for the modified G-SPSA algorithm:

(C1) there exists a compact stability region $\mathbf{S} \subseteq \mathbf{R}^{n}$ for $\dot{x}(t)=-\nabla f(x(t))$ (which is nonzero-measure and for which $\dot{x}(t)=-\nabla f(x(t))$ with $x(0) \in \mathbf{S}$ results in $x(\infty)=x^{*}$ ) such that $\hat{x}_{k} \in \mathbf{S}$ occurs infinitely often for every $x_{0} \in \mathbf{S}$ and almost all sample points;

(C2) $\sup _{k \in \mathbf{N}}\left\|\hat{x}_{k}\right\|<\infty$ holds w.p. 1 for every $x_{0} \in \mathbf{S}$; (C3) there exists a $\gamma_{4} \in \mathbf{R}_{+}$such that $E\left[f\left(\hat{x}_{k}+\right.\right.$ $\left.\left.c_{k 1} T_{k} \xi_{k}\right)^{2}\right] \leq \gamma_{4}$ and $E\left[f\left(\hat{x}_{k}-c_{k 2} T_{k} \xi_{k}\right)^{2}\right] \leq \gamma_{4}$ for hold, then every $x_{0} \in \mathbf{S}$ and $k \in \mathbf{N}$;

$$
\lim _{k \rightarrow \infty} x_{k}=x^{*}
$$

holds w.p. 1 for every $x_{0} \in \mathbf{S}$.

Proof: See Appendix II.

From Proposition 2, it turns out that a local solution to the problem in (11) is given by the G-SPSA algorithm under several conditions. In particular, it should be noted that the conditions for the convergence do not heavily depend on the information of the uncertain matrix set $\mathbf{T}$ as seen in (A3) and (A6) and thus this result is useful for the case where we do not have precise information about $\mathbf{T}$ but have an estimation $\hat{\mathbf{T}}$ of $\mathbf{T}$.

Most of the conditions in Proposition 2 are fairly standard in stochastic approximation [21], [25]. Conditions (A1)-(A6) are concerned with the function $f$, the noise $\varepsilon_{k+}, \varepsilon_{k-}$, and the uncertain time-varying matrix $T_{k}$. (A1) means that $f$ is smooth enough and (A4) is common for descent-type algorithms, saying that $x^{*}$ is a local minimum point of $f$. (A2) resembles the common martingale difference noise assumption appearing in standard stochastic approximation algorithms (see, e.g., [30]). This plays an important role to make the search direction $d\left(x_{k}, c_{k}, \xi_{k}, \delta_{k}\right)$ be a gradient approximation as in (12) and to prove that a partial sum process associated with the difference $d\left(x_{k}, c_{k}, \xi_{k}, \delta_{k}\right)-E\left[d\left(x_{k}, c_{k}, \xi_{k}, \delta_{k}\right) \mid x_{k}\right]$ is martingale (see the proof of Proposition 2). In our source seeking problem, the condition holds if the field switches sufficiently fast. (A5) prescribes the second-order moment of the noise terms. (A3) implies $T_{0} T_{0}^{\top}=T_{1} T_{1}^{\top}=\cdots=T_{\infty} T_{\infty}^{\top}=I$ and (A6) is a technical assumption to prove the convergence in probability by reducing the G-SPSA algorithm into the Robbins-Monro Algorithm in Appendix II-A. Next, (B1)-(B4) are imposed for the parameters designed by the users, and they will be a guideline to design. A typical parameter choice of $\left\{a_{k}\right\},\left\{c_{k 1}\right\}$, and $\left\{c_{k 2}\right\}$ is

$$
a_{k}:=\frac{a}{(k+1)^{\alpha}}, \quad c_{k 1}:=\frac{c}{(k+1)^{\gamma}}, \quad c_{k 2}:=c_{k 1}
$$

where $a, \alpha, c, \gamma \in \mathbf{R}_{+}$are arbitrarily given so that $\alpha \leq 1$ and $\alpha-\gamma>0.5$. It may be reasonable to set large numbers to $a_{k}, c_{k 1}$, and $c_{k 2}$ in the initial phase and let them be gradually smaller with $k$ so as to search the minimizer roughly at the beginning and search it precisely near the minimizer. On the other hand, a typical probability distribution $\Xi_{k}$ of $\xi_{k}$ is based on the elementwise Bernoulli trial with outcome \pm 1 and equal probabilities (i.e., $\xi_{k i}=-1$ or $\xi_{k i}=1$ occurs the same probability). The condition that $\Xi_{k}$ be Bernoulli-type is not necessary but it has been proven in [26] that the Bernoulli type is optimal in many cases. Other possible choices are found in [27]. The last $(\mathrm{C} 1)-(\mathrm{C} 3)$ are technical conditions to guarantee the convergence. $(\mathrm{C} 1)$ and $(\mathrm{C} 2)$ are challenging to check, but it is known that they are not restrictive conditions in practice, as addressed in [21] and [25]. This fact has been demonstrated by a number of examples (a great list of the existing results is provided in [28]). In addition, it has been explained in [25] that these can be ignored by replacing the algorithm in (9) with a projected version (like the projected gradient method for constrained optimization problems). A projected version is given by $x_{k+1}=\Pi_{\mathbf{X}}\left(x_{k}-a_{k} d\left(x_{k}, c_{k}, \xi_{k}, \delta_{k}\right)\right)$ where $\mathbf{X} \subset \mathbf{R}^{n}$ is a closed hyperrectangle in which $x_{k}$ has to be constrained and $\Pi_{\mathbf{X}}: \mathbf{R}^{n} \rightarrow \mathbf{R}^{n}$ is the projection onto the set $\mathbf{X}$. Meanwhile, it should be noted that (C1) holds if $f$ is convex and (C2) holds, and a simple sufficient condition ${ }^{2}$ for $(\mathrm{C} 2)$ is given in [30]. Also, a weaker condition of $(\mathrm{C} 2)$, which is for a more general class of stochastic approximation algorithms, has been given in [29]. (C3) is related to the second-order moment of $f$ with randomly perturbed inputs and is not so restrictive if (C2) holds.

${ }^{2}(\mathrm{C} 2)$ holds if 1) $\nabla f(x)$ is Lipschitz, 2) the conditions in Proposition 1 hold, 3) the first and second conditions of (B2) hold, and 4) for $\nabla f_{c}(x):=\nabla f(c x) / c, \nabla f_{c}(x) \rightarrow \nabla f_{\infty}(x)$ as $c \rightarrow \infty$ and $\dot{x}=\nabla f_{\infty}(x)$ has the origin as its unique globally asymptotically stable equilibrium. This is just a sufficient condition for (C2) (i.e., (C2) covers more cases) but may be the most practical for directly checking (C2). See [30] for further details. 
Similar to the original SPSA algorithm [21], the G-SPSA algorithm has the following two features. First, the algorithm solves the problem in (11) when neither $f$ nor $\nabla f$ is known, and instead, only noisy measurements of $f$ are available. In fact, it can be seen that the search direction $d\left(x_{k}, c_{k}, \xi_{k}, \delta_{k}\right)$ in (10) contains the noisy measurements $f\left(x_{k}+c_{k 1} T_{k} \xi_{k}\right)+\varepsilon_{k+}$ and $f\left(x_{k}-c_{k 2} T_{k} \xi_{k}\right)+\varepsilon_{k-}$. Next, the number of measurements to determine the search direction is only two and is independent of the dimension $n$ of the variable $x$. This merit can be understood by the fact that the number of measurements for the difference approximation of $\nabla f\left(=\left[\partial f(x) / \partial x_{1} \cdots \partial f(x) / \partial x_{n}\right]^{\top}\right)$ grows with $n$.

The proposed algorithm is equivalent to the original SPSA algorithm in [21] if

$$
c_{k 1} \equiv c_{k 2}, \quad \mathbf{T}=\left\{I_{n}\right\}
$$

for (10). Basically, the proposed algorithm is an extention by the coordinate transformation, while the following differences should be stressed:

- the search direction (10) is based on unequal two-sided perturbations $c_{k 1} T_{k} \xi_{k} \neq c_{k 2} T_{k} \xi_{k}$, while the original version is of equal perturbations $c_{k 1} T_{k} \xi_{k}=c_{k 2} T_{k} \xi_{k}$;

- the direction (10) is regarded as a time-varying function of $x_{k}, c_{k 1}, c_{k 2}, \xi_{k}, \varepsilon_{k+}$, and $\varepsilon_{k-}$ (by the time-dependent matrices $T_{k}$ ) unlike the time-invariant original one.

These properties play a key role for solving the source seeking problem. Note that, though one may consider that the former is just an excess of generality, (12) is not straightforwardly obtained by [21] in the case where $c_{k 1} T_{k} \xi_{k} \neq c_{k 2} T_{k} \xi_{k}$, because we need a different formula [(40) in Appendix I-B] to derive it. Note also that the latter, i.e., the idea of the time-varying coordinate transformation by $T_{k}$, is not standard in static optimization methods. These imply that the proposed algorithm will be a special technique for the source seeking by mobile robots.

\section{B. G-SPSA Based Stochastic Discrete Trajectories for Source Seeking}

Based on the G-SPSA algorithm, we provide a set of stochastic discrete trajectories for source seeking.

For the signal field $S$, let us introduce the random variable

$$
\varepsilon(t):=f_{\sigma(t)}(x(t))-E\left[f_{\sigma(t)}(x(t)) \mid x(t)\right] .
$$

Its conditional expected value is zero, i.e.,

$$
E[\varepsilon(t) \mid x(t)]=0 \quad \forall(t, x(t)) \in \mathbf{R}_{0+} \times \mathbf{R}^{n_{1}} .
$$

By (3) and (15), $S$ is expressed as

$$
y(t)=E\left[f_{\sigma(t)}(x(t)) \mid x(t)\right]+\varepsilon(t) .
$$

Then by respectively regarding $x(t), E\left[f_{\sigma(t)}(x(t)) \mid x(t)\right]$, and $\varepsilon(t)$ as $x_{k}, f(x)$, and $\varepsilon_{k \pm}$ in the G-SPSA algorithm, we obtain the following result.
Theorem 1: For the signal field $S$, assume that there exists a source $x^{*} \in \mathbf{R}^{n_{1}}$. Suppose that the uncertain matrix set $\mathbf{T} \subseteq \mathbf{R}^{n_{1} \times n_{1}}$ and the design parameters $\left\{a_{k}\right\},\left\{c_{k 1}\right\},\left\{c_{k 2}\right\}$, $\left\{\Xi_{k}\right\}$ of the G-SPSA algorithm are given so as to satisfy (A3), (A6), and (B1)-(B4) (note that (A1), (A2), (A4), and (A5) automatically hold, which will be shown in the proof), and let $\left\{x_{01}, x_{02}, x_{10}, x_{11}, x_{12}, x_{20}, x_{21}, x_{22}, \ldots\right\}$ be the stochastic process given by

$$
\left\{\begin{array}{l}
x_{k 1}=x_{k 0}+c_{k 1} T_{k} \xi_{k} \\
x_{k 2}=x_{k 0}-c_{k 2} T_{k} \xi_{k} \\
x_{(k+1) 0}=x_{k 0}-a_{k} T_{k} \hat{d}\left(y_{k 1}, y_{k 2},\left(c_{k 1}+c_{k 2}\right), \xi_{k}\right)
\end{array}\right.
$$

where $x_{00} \in \mathbf{R}^{n_{1}}$ is the initial state, $T_{k} \in \mathbf{T}$ is the uncertain time-varying matrix:

$$
\begin{aligned}
\hat{d}\left(y_{k 1}, y_{k 2},\left(c_{k 1}+c_{k 2}\right), \xi_{k}\right) & :=\frac{y_{k 1}-y_{k 2}}{\left(c_{k 1}+c_{k 2}\right)} \xi_{k}^{(-1)} \\
y_{k i}: & =f_{\sigma_{k i}}\left(x_{k i}\right)
\end{aligned}
$$

and $\sigma_{k i}$ are the i.i.d. random variables from the probability distribution $g$ in Section II-A. If (C1)-(C3) hold for the modified G-SPSA algorithm with $f(x):=E\left[f_{\sigma}(x) \mid x\right], \varepsilon_{k+}:=$ $f_{\sigma_{k 1}}(x)-E\left[f_{\sigma}(x) \mid x\right], \varepsilon_{k-}:=f_{\sigma_{k 2}}(x)-E\left[f_{\sigma}(x) \mid x\right]$, some $\hat{\mathbf{T}} \subseteq \mathbf{R}^{n_{1} \times n_{1}}$ including $\mathbf{T},\left\{a_{k}\right\},\left\{c_{k 1}\right\},\left\{c_{k 2}\right\},\left\{\Xi_{k}\right\}, x_{00}$, and $x^{*}$, then

$$
\lim _{k \rightarrow \infty} x_{k i}=x^{*} \quad\left(\forall\left\{T_{k}\right\} \in \prod_{k=0}^{\infty} \mathbf{T}\right)
$$

w.p.1 for every $x_{00} \in \mathbf{S}$ and $i \in\{0,1,2\}$ [where $\mathbf{S}$ is given in (C1)].

Proof: For $f(x):=E\left[f_{\sigma}(x) \mid x\right]$ and $\varepsilon_{k i}:=f_{\sigma_{k i}}\left(x_{k i}\right)-$ $E\left[f_{\sigma}\left(x_{k i}\right) \mid x_{k i}\right](i=1,2)$, we have $y_{k i}=f\left(x_{k i}\right)+\varepsilon_{k i}$ in a similar way to (17). This and the first two equations of (18) provide $y_{k 1}=f\left(x_{k 0}+c_{k 1} T_{k} \xi_{k}\right)+\varepsilon_{k 1}$ and $y_{k 2}=f\left(x_{k 0}-c_{k 2} T_{k} \xi_{k}\right)+$ $\varepsilon_{k 2}$. So it follows under $x_{k}=x_{k 0}, \varepsilon_{k+}=\varepsilon_{k 1}$, and $\varepsilon_{k-}=\varepsilon_{k 2}$ that the third equation of (18) is equivalent to a G-SPSA algorithm. Then, (A3), (A6), (B1)-(B4), and (C1)-(C3) hold for the G-SPSA algorithm as stated, and also (A1), (A2), (A4), and (A5) hold by the thrice differentiability of $f_{\sigma}$, the i.i.d. sample condition for $\sigma_{k i}$ (which implies that $x_{k i}$ is independent of $\left.\left\{\sigma_{k i}, \sigma_{(k+1) i}, \ldots\right\}\right)$, the definitions of $\varepsilon_{k i}$ and the source, (C2), and (16). So it turns out from Proposition 2 that (21) with $i=0$ holds for every $x_{00} \in \mathbf{S}$. Moreover, since $\xi_{k}$ and $T_{k}$ are bounded as stated in (B1) (a) and (A3), the first two equations of (18) and (B2) imply $\lim _{k \rightarrow \infty}\left\|x_{k i}-x_{k 0}\right\|=0$ for $i \in\{1,2\}$. This and (21) for $i=0$ prove that (21) with $i \in\{1,2\}$ holds for every $x_{00} \in \mathbf{S}$.

Theorem 1 presents a set of stochastic trajectories converging to a source almost surely (in which each trajectory is specified by $\left\{a_{k}\right\},\left\{c_{k 1}\right\},\left\{c_{k 2}\right\}$, and $\left\{\Xi_{k}\right\}$ ). The trajectories are given by fully exploiting the advantages of the G-SPSA algorithm: they only use the measurements $y_{k i}(i=1,2)$ of $f_{\sigma_{k i}}$ for finding a source, and the measurements to determine the search direction are collected by the only two auxiliary movements to the positions $x_{k 1}$ and $x_{k 2}$. 


\section{Stochastic Source SeEking Controllers}

\section{A. Source Seeking Controllers in a General Form}

Now, we derive a controller $K$ which sequentially generates the next waypoints as a part of a stochastic trajectory in the form of (18) and steers the robot $P$ along the trajectory.

Since $P$ does not have the position information in the world coordinate frame, $P$ cannot follow some trajectories in the form of (18). So, in order to obtain an appropriate trajectory in the sensor-free situation, we transform the condition (18) into that in the body fixed frame $\Sigma\left(t_{k 0}\right)$ :

$$
\left\{\begin{array}{l}
z_{k 1}=c_{k 1} R_{n_{1}}\left(-\theta\left(t_{k 0}\right)\right) T_{k} \xi_{k} \\
z_{k 2}=-c_{k 2} R_{n_{1}}\left(-\theta\left(t_{k 0}\right)\right) T_{k} \xi_{k} \\
z_{(k+1) 0}=-a_{k} R_{n_{1}}\left(-\theta\left(t_{k 0}\right)\right) T_{k} \hat{d}\left(y_{k 1}, y_{k 2},\left(c_{k 1}+c_{k 2}\right), \xi_{k}\right)
\end{array}\right.
$$

where $x\left(t_{k 0}\right)=x_{k 0}$ and $\theta\left(t_{k 0}\right)$ is the rotational position of $P$ at time $t_{k 0}$. Here, if $c_{k 1}=0$ and $T_{k}=R_{n_{1}}\left(\theta\left(t_{k 0}\right)\right)(k=$ $1,2, \ldots)$, we have $z_{k 1}=0, z_{k 2}=-c_{k 2} \xi_{k}$, and $z_{(k+1) 0}=$ $-a_{k} \hat{d}\left(y_{k 1}, y_{k 2}, c_{k 2}, \xi_{k}\right)$. This is a condition excluding the position information of $\theta\left(t_{k 0}\right)$ in the world coordinate frame and thus is suitable in the sensor-free situation. This idea provides a solution to Problem 1.

Theorem 2: For the feedback system in Fig. 2, assume that (P1) holds for $\Delta \tau \in \mathbf{R}_{+}$and $\left(r_{1}, r_{2}, r_{3}\right) \in \mathbf{R}_{0+}^{3}$ and there exists a source $x^{*} \in \mathbf{R}^{n_{1}}$. Let $t_{00}:=0$ and suppose that the tuning parameters:

- the discrete time sequence $\left\{t_{02}, t_{10}, t_{12}, \ldots\right\}$ such that $t_{k 2}-t_{k 0}>\max \{\eta, \Delta \tau\}$ and $t_{(k+1) 0}-t_{k 2}>\max \{\eta, \Delta \tau\}$ for every $k \in\{0,1, \ldots\}$;

- $\left\{a_{k}\right\},\left\{c_{k 2}\right\}$, and $\left\{\Xi_{k}\right\}$ satisfying (B1)-(B4) under the condition $c_{k 1} \equiv 0$;

- the positive integer $N$ and the desirable rotation angle $\Delta \psi_{k i} \in \boldsymbol{\Phi}_{N}(k=0,1, \ldots, \infty, i=0,2)$;

are given, where $\boldsymbol{\Phi}_{N}:=\{0, \pm \pi / N, \pm 2 \pi / N, \ldots, \pm(N-$ 1) $\pi / N, \pi\}^{n_{2}}$ (e.g., $\boldsymbol{\Phi}_{3}=\{-2 \pi / 3,-\pi / 3,0, \pi / 3,2 \pi / 3, \pi\}^{n_{2}}$ ). If (C1)-(C3) hold for the modified G-SPSA algorithm with the same $f, \varepsilon_{k+}, \varepsilon_{k-}$ as in Theorem $1, \hat{\mathbf{T}}:=\left\{L \in \mathbf{R}^{n_{1} \times n_{1}} \mid \exists \varphi \in\right.$ $[-\pi, \pi)^{n_{2}}$ s.t. $\left.L=R_{n_{1}}(\varphi)\right\}$ (the set of $n_{1}$-dimensional rotation matrices), $\left\{a_{k}\right\}, c_{k 1} \equiv 0,\left\{c_{k 2}\right\},\left\{\Xi_{k}\right\}, x_{0}:=x(0)$, and $x^{*}$, then the controller $K$ satisfying (23), shown at the bottom of the page, for $k=0,1, \ldots$ is a solution to Problem 1 for any $r>r_{2} \pi \sqrt{n_{2}}+r_{3}$.

Proof: Let $x(t)$ (also $x\left(t_{k i}\right)$ ) denote the translational position of the robot $P$ for the controller $K$ satisfying (23). Then this theorem is a direct consequence of the following four facts:

1) The set $\mathbf{S}$, which is given by $(\mathrm{C} 1)$, is nonzero-measure and includes the source $x^{*}$ in its interior.
2) For every $\left(x_{0}, t\right) \in \mathbf{S} \times \mathbf{R}_{0+},\|x(t)\|<\infty$ w.p.1 under $x(0)=x_{0}$.

3) For every $x_{0} \in \mathbf{S}$ and $i \in\{0,2\}, \lim _{k \rightarrow \infty} x\left(t_{k i}\right)=x^{*}$ w.p.1 under $x(0)=x_{0}$.

4) For each $x_{0} \in \mathbf{S}$ and $\nu \in \mathbf{R}_{+}$, there exists a $k_{\text {min }} \in \mathbf{N}$ such that the following two conditions hold for every $k \in\left\{k_{\text {min }}, k_{\text {min }}+1, \ldots\right\}:$ a) $\left\|x(t)-x\left(t_{k 0}\right)\right\| \leq$ $\nu+r_{2} \pi \sqrt{n_{2}}+r_{3}$ for every $t \in\left[t_{k 0}, t_{k 2}\right]$, b) $\| x(t)-$ $x\left(t_{k 2}\right) \| \leq \nu+r_{2} \pi \sqrt{n_{2}}+r_{3}$ for every $t \in\left[t_{k 2}, t_{(k+1) 0}\right]$.

Fact 1) is trivial. So we next prove the other facts in the order of 3$), 2$ ), and 4).

3): By the definition of the point-to-point control input $v$ [see (6) and (7)], the controller $K$ steers the robot $P$ as

$$
\begin{aligned}
x\left(t_{k 2}\right)= & x\left(t_{k 0}\right)-R_{n_{1}}\left(\theta\left(t_{k 0}\right)\right)\left(c_{k 2} \xi_{k}\right) \\
x\left(t_{(k+1) 0}\right)= & x\left(t_{k 2}\right)+R_{n_{1}}\left(\theta\left(t_{k 2}\right)\right) R_{n_{1}}\left(-\Delta \psi_{k 0}\right) \\
& \times\left(c_{k 2} \xi_{k}-a_{k} \hat{d}\left(y\left(t_{k 0}\right), y\left(t_{k 2}\right), c_{k 2}, \xi_{k}\right)\right) \\
= & x\left(t_{k 0}\right)-a_{k} R_{n_{1}}\left(\theta\left(t_{k 0}\right)\right) \hat{d}\left(y\left(t_{k 0}\right), y\left(t_{k 2}\right), c_{k 2}, \xi_{k}\right) .
\end{aligned}
$$

Then, the conditions on the discrete time sequence, especially, $t_{k 2}-t_{k 0}>\eta$ and $t_{(k+1) 0}-t_{k 2}>\eta$, imply that the corresponding noise signals $\varepsilon_{0+}, \varepsilon_{0-}, \varepsilon_{1+}, \varepsilon_{1-}, \ldots$ are not correlated and so (A2) holds. Thus it turns out from (3) and (20) that the stochastic process $\left\{x\left(t_{00}\right), x\left(t_{02}\right), x\left(t_{10}\right), x\left(t_{10}\right), x\left(t_{12}\right), \ldots\right\}$ is equivalent to that in Theorem 1 with $\mathbf{T}:=\{L \in$ $\mathbf{R}^{n_{1} \times n_{1}} \mid \exists \varphi \in\left\{\theta\left(t_{k 0}\right)\right\} \oplus \boldsymbol{\Phi}_{N}$ s.t. $\left.L=R_{n_{1}}(\varphi)\right\},\left\{a_{k}\right\}$, $c_{k 1} \equiv 0,\left\{c_{k 2}\right\}$, and $\left\{\Xi_{k}\right\}$, and it is obvious that (A3) and (A6) hold for $\mathbf{T}$. In addition, (B1)-(B4) hold for the given $\left\{a_{k}\right\}$, $\left\{c_{k 2}\right\},\left\{\Xi_{k}\right\}$, and $c_{k 1} \equiv 0$, and (C1)-(C3) hold for the modified G-SPSA algorithm as stated. So 3 ) follows from Theorem 1.

2): Suppose that $x_{0} \in \mathbf{S}$ is given and let $x(0)=x_{0}$. From (23) and the definition of $v$, we have $\|z(t)\| \leq r_{1}\left\|c_{k 2} \xi_{k}\right\|+$ $r_{2}\left\|\Delta \psi_{k 0}\right\|+r_{3}$ for $t \in\left[t_{k 0}, t_{k 2}\right]$ and

$$
\begin{aligned}
& \left\|z(t)+c_{k 2} \xi_{k}\right\| \\
& \leq r_{1}\left\|R_{n_{1}}\left(-\Delta \psi_{k 0}\right)\left(c_{k 2} \xi_{k}-a_{k} \hat{d}\left(y\left(t_{k 0}\right), y\left(t_{k 2}\right), c_{k 2}, \xi_{k}\right)\right)\right\| \\
& \quad+r_{2}\left\|\Delta \psi_{k 2}\right\|+r_{3} \\
& =r_{1}\left\|c_{k 2} \xi_{k}-a_{k} \hat{d}\left(y\left(t_{k 0}\right), y\left(t_{k 2}\right), c_{k 2}, \xi_{k}\right)\right\|+r_{2}\left\|\Delta \psi_{k 2}\right\|+r_{3}
\end{aligned}
$$

for $t \in\left[t_{k 2}, t_{(k+1) 0}\right]$ in the body fixed coordinate frame $\Sigma\left(t_{k 0}\right)$, which are rewritten as

$$
\begin{aligned}
\left\|x(t)-x\left(t_{k 0}\right)\right\| \leq & r_{1}\left\|c_{k 2} \xi_{k}\right\|+r_{2}\left\|\Delta \psi_{k 0}\right\|+r_{3} \\
& \left(\forall t \in\left[t_{k 0}, t_{k 2}\right]\right) \\
\left\|x(t)-x\left(t_{k 2}\right)\right\| \leq & r_{1}\left\|c_{k 2} \xi_{k}-a_{k} \hat{d}\left(y\left(t_{k 0}\right), y\left(t_{k 2}\right), c_{k 2}, \xi_{k}\right)\right\| \\
& +r_{2}\left\|\Delta \psi_{k 2}\right\|+r_{3} \quad\left(\forall t \in\left[t_{k 2}, t_{(k+1) 0}\right]\right)
\end{aligned}
$$

$$
u(t)= \begin{cases}v\left(t, t_{k 2}-t_{k 0}, 0 \rightarrow\left[\begin{array}{c}
-c_{k 2} \xi_{k} \\
\Delta \psi_{k 0}
\end{array}\right], r_{1}, r_{2}, r_{3}\right), & \text { if } t \in\left[t_{k 0}, t_{k 2}\right] \\
v\left(t, t_{(k+1) 0}-t_{k 2}, 0 \rightarrow\left[\begin{array}{c}
R_{n_{1}}\left(-\Delta \psi_{k 0}\right)\left(c_{k 2} \xi_{k}-a_{k} \hat{d}\left(y\left(t_{k 0}\right), y\left(t_{k 2}\right), c_{k 2}, \xi_{k}\right)\right) \\
\Delta \psi_{k 2}
\end{array}\right], r_{1}, r_{2}, r_{3}\right), & \text { if } t \in\left[t_{k 2}, t_{(k+1) 0}\right]\end{cases}
$$


in the world coordinate frame [see (8)]. Then,

$$
\left\|\Delta \psi_{k i}\right\| \leq \pi \sqrt{n_{2}}
$$

by the definition of $\boldsymbol{\Phi}_{N}$ (which implies $\Delta \psi_{k i} \in[-\pi, \pi)^{n_{2}}$ ). Furthermore, as shown in the proof of 3 ), the stochastic process $\left\{x\left(t_{00}\right), x\left(t_{02}\right), x\left(t_{10}\right), x\left(t_{10}\right), x\left(t_{12}\right), \ldots\right\}$ is equivalent to that in Theorem 1, which, together with (B1) and (B2), implies that $x\left(t_{k i}\right), c_{k 2} \xi_{k}$, and $a_{k} \hat{d}\left(y\left(t_{k 0}\right), y\left(t_{k 2}\right), c_{k 2}, \xi_{k}\right)(k=0,1, \ldots$, $i=0,1,2)$ are bounded w.p.1. So we have 2).

4): Applying (24), (25), and (28) to (26) and (27) gives

$$
\begin{aligned}
\left\|x(t)-x\left(t_{k 0}\right)\right\| \leq & r_{1}\left\|x\left(t_{k 2}\right)-x\left(t_{k 0}\right)\right\|+r_{2} \pi \sqrt{n_{2}}+r_{3} \\
& \left(\forall t \in\left[t_{k 0}, t_{k 2}\right]\right) \\
\left\|x(t)-x\left(t_{k 2}\right)\right\| \leq & r_{1}\left\|x\left(t_{(k+1) 0}\right)-x\left(t_{k 2}\right)\right\|+r_{2} \pi \sqrt{n_{2}}+r_{3} \\
& \left(\forall t \in\left[t_{k 2}, t_{(k+1) 0}\right]\right) .
\end{aligned}
$$

Then 3) implies that, for each $x_{0} \in \mathbf{S}$ and $\nu \in \mathbf{R}_{+}$, there exists a $k_{\text {min }} \in \mathbf{N}$ such that $\left\|x\left(t_{k 2}\right)-x\left(t_{k 0}\right)\right\| \leq \nu / r_{1}$ and $\left\|x\left(t_{(k+1) 0}\right)-x\left(t_{k 2}\right)\right\| \leq \nu / r_{1}$ for every $k \in\left\{k_{\text {min }}, k_{\text {min }}+\right.$ $1, \ldots\}$ w.p.1. This completes the proof of 4$)$.

Theorem 2 presents stochastic source seeking controllers composed of two point-to-point controllers. The controllers is given by the G-SPSA algorithm and it executes the G-SPSA algorithm step by step: after $P$ obtains a measurement at the current position, the first input $v$ moves $P$ to a point to collect another measurement and the second input $v$ steers $P$ to a point indicated by the search direction, which is iterated. Then the values about the mobility of $P$, i.e., $\Delta \tau$ and $\left(r_{1}, r_{2}, r_{3}\right)$, are related to the convergence property: $\Delta \tau$ corresponds to the possible convergence speed as shown in the condition for the discrete time sequence $\left\{t_{02}, t_{10}, t_{12}, \ldots\right\}$, while $\left(r_{1}, r_{2}, r_{3}\right)$ has the relation to the smoothness of the paths connecting the waypoints given in Theorem 1 , and also $r_{2}$ and $r_{3}$ are numbers characterizing the radius $r$ of the closed ball to which $P$ converges.

Remark 1: It is assumed in Theorem 2 that $t_{k 2}-t_{k 0}>$ $\max \{\eta, \Delta \tau\}$ and $t_{(k+1) 0}-t_{k 2}>\max \{\eta, \Delta \tau\}$. The condition on $\Delta \tau$ is related to the mobility of the robot $P$. On the other hand, the condition on $\eta$ is reasonable in finding the source of the "expected" signal field. In fact, in our source seeking method, the source is sought by sampling the value of the stochastically switching signal field, and then the condition guarantees that the number of samples is large. If $\eta$ is greater than $t_{k 2}-t_{k 0}$ or $t_{(k+1) 0}-t_{k 2}$, it is a practical option that the discrete time sequence $\left\{t_{02}, t_{10}, t_{12}, \ldots\right\}$ is reset so as to satisfy the condition. Note that the control designer usually has the flexibility in choosing the time sequence.

Remark 2: It should be clarified why the original SPSA algorithm [21], given by (9), (10), and (14), is not suitable for our source seeking problem. First, the discrete trajectories given by the original SPSA algorithm cannot be followed by $P$ in practice. In fact, (14) and (18) specify a robot moving trajectory in the body fixed frame $\Sigma\left(t_{k 0}\right)$ [see (5)] as

$$
\left\{\begin{array}{l}
z_{k 1}=c_{k 1} R_{n_{1}}\left(-\theta\left(t_{k 0}\right)\right) \xi_{k} \\
z_{k 2}=-c_{k 1} R_{n_{1}}\left(-\theta\left(t_{k 0}\right)\right) \xi_{k} \\
z_{(k+1) 0}=-a_{k} R_{n_{1}}\left(-\theta\left(t_{k 0}\right)\right) \hat{d}\left(y_{k 1}, y_{k 2}, 2 c_{k 1}, \xi_{k}\right)
\end{array}\right.
$$

in which the three destinations depend on the position $\theta\left(t_{k 0}\right)$ in the world coordinate frame. However, the position information is not available in $K$ as shown in Fig. 2. Thus, in general, it is impossible to move $P$ along the trajectory. The only case that $P$ can follow the trajectory is when the trajectory is given based on the coordinate frame whose origin is $(x(0), \theta(0))$ and a feedforward estimator, that is, a solver of the differential equation (1) with the initial state and the input history, is used to estimate the robot position on the time interval $[0, \infty)$. In this case, the rotational position in the frame can be estimated only with the model of (1), the zero initial position, and the input history. However, it is obvious that such feedforward estimation produces an accumulative error in the real world and is unrealistic. Second, unlike the proposed controllers in (23), $K$ has to be a feedforward motion controller driving the robot $P$ to three points. That is, as seen in (29), to execute one step of the SPSA algorithm, $P$ has to visit two points to collect measurements and move to a point indicated by the search direction. Then, in our problem, this movement must be achieved without using the position information. Clearly, in the presence of uncertainty, such three-point feedforward control is less desirable than the two-point feedforward control in (23).

Remark 3: As a variant of the SPSA algorithm [21], the onemeasurement type, which requires the only one measurement to determine the search direction, has been developed in [31]. This can be also extended to an appropriate form for the source seeking. However, as pointed out in [31], the one-measurement algorithm is less efficient in many cases.

Remark 4: The controllers proposed in Theorem 2 are given without explicitly considering the mobility of the robot $P$. This implies that the controllers may lead to some unnatural behavior. On the other hand, as shown in the next subsection, if $P$ is the unicycle robot, the resulting movement is similar (not completely consistent) to a well-known biological phenomenon, the bacterial chemotaxis, that is, the phenomenon that bacteria in an environment sense a chemical concentration and move to a more favorable position [32]. In fact, it is known that the mobility of the bacteria is the almost same as that of the unicycle and the movement of the chemotaxis is composed of a random turn and a forward move. Meanwhile, the proposed controller let the unicycle robot $P$ perform the random turn and the feedforward/backward move. In this sense, the movement by the proposed controllers may not be unnatural for some class of $P$. In relation to this, it should be remarked that a source seeking method that mimics the bacterial chemotaxis has been proposed in [6].

Remark 5: Though the boundedness and the convergence of $x(t)$ is guaranteed for the proposed controller, $P$ might drift far away on the way to the source even when the robot $P$ starts near the source. This is because the gains $a_{k}$, which are relative to the moving distance on the time intervals $\left[t_{k 0}, t_{(k+1) 0}\right]$, have to be large numbers in the early iterations to avoid the sluggish performance and so the value of $E\left[f_{\sigma\left(t_{k 0}\right)}\left(x\left(t_{k 0}\right)\right) \mid x\left(t_{k 0}\right)\right]$ does not always decrease even if the initial position $x(0)$ is near the source. In exchange for the undesirable transient, such a policy contributes to good performance for $x(0)$ far from the source. Generally, in the optimization using a gradient-free descent 
method (such as the finite-difference approximation), such a phenomenon is unavoidable because the step size sequence which guarantees monotonically decreasing behavior cannot be determined without the information of the gradient. These points imply that the possibility of the drift is a theoretical limitation of our method. However, it should be noted that our method yields a certain result for switching fields despite of such a limitation.

\section{B. Source Seeking Controllers for Some Specific Robots}

Next, we focus on two- and three-dimensional nonholonomic robots and show that there exist very simple source seeking controllers.

1) 2-D Nonholonomic Robot Case: We first prepare the following result.

Lemma 1: Consider the search direction $d\left(x_{k}, c_{k}, \xi_{k}, \delta_{k}\right)$ in (10). If $\xi_{k} \in\{-\rho, \rho\}^{n}$ for $\rho \in \mathbf{R}_{+}$, then the vectors $T_{k} \xi_{k}$ and $d\left(x_{k}, c_{k}, \xi_{k}, \delta_{k}\right)$ are linearly dependent. More precisely, the relation

$$
d\left(x_{k}, c_{k}, \xi_{k}, \delta_{k}\right)=\left(\omega_{k} \rho^{-2}\right) T_{k} \xi_{k}
$$

holds for the scalar

$$
\omega_{k}:=\frac{\left(f\left(x_{k}+c_{k 1} T_{k} \xi_{k}\right)+\varepsilon_{k+}\right)-\left(f\left(x_{k}-c_{k 2} T_{k} \xi_{k}\right)+\varepsilon_{k-}\right)}{\left(c_{k 1}+c_{k 2}\right)}
$$

Proof: For $\xi_{k} \in\{-\rho, \rho\}^{n}$, we have $\xi_{k}=\rho \operatorname{sign}\left(\xi_{k}\right)$. So it follows that $T_{k} \xi_{k}=\rho T_{k} \operatorname{sign}\left(\xi_{k}\right)$ and

$$
\begin{aligned}
d\left(x_{k}, c_{k}, \xi_{k}, \delta_{k}\right) & =\omega_{k} T_{k} \xi_{k}^{(-1)}=\rho^{-1} \omega_{k} T_{k}\left(\operatorname{sign}\left(\xi_{k}\right)\right)^{(-1)} \\
& =\rho^{-1} \omega_{k} T_{k} \operatorname{sign}\left(\xi_{k}\right)
\end{aligned}
$$

This proves (30).

Lemma 1 shows that, if $\Xi_{k}(i=0,1, \ldots)$ are the probability distributions based on the elementwise Bernoulli trial shown in Section III-A, the vectors in (18), $x_{k 1}, x_{k 2}$, and $x_{(k+1) 0}$, are on a line segment in $\mathbf{R}^{n_{1}}$. So to execute one step of the G-SPSA algorithm, it is enough to drive $P$ on a line segment. This property presents a very simple controller for the nonholonomic unicycle (2).
Theorem 3: For the feedback system in Fig. 2, assume that $P$ is the unicycle (2) and there exists a source $x^{*} \in \mathbf{R}^{2}$. Let $K$ be the discrete-time controller with the zero-order hold:

$$
\left\{\begin{array}{l}
y_{\text {pre }}[s+1]=y[s], \\
u[s]=\left\{\begin{array}{ll}
{\left[\begin{array}{c}
0 \\
\frac{1}{h} \zeta[s]
\end{array}\right],} & \text { if } s=0,3,6, \ldots \\
{\left[\begin{array}{c}
-\frac{\lambda}{h} c_{\lfloor s / 3\rfloor 2} \\
0
\end{array}\right],} & \text { if } s=1,4,7, \ldots \\
{\left[\frac{\lambda}{h}\left(c_{\lfloor s / 3\rfloor 2}-a_{\lfloor s / 3\rfloor} \frac{y_{p r e}[s]-y[s]}{c_{\lfloor s / 3\rfloor 2}}\right)\right],} & \text { if } s=2,5,8, \ldots
\end{array}\right\}
\end{array}\right.
$$

where $h \in(\eta, \infty)$ is the sampling period, $s \in \mathbf{N}$ is the discrete time $(s=\lfloor t / h\rfloor), y_{\text {pre }}[s] \in \mathbf{R}$ is the state to save $y[s-1]$ $(=y((s-1) h)), \zeta[s] \in \mathbf{R}$ is the i.i.d. random variable drawn from the uniform distribution on

$$
\left\{\frac{\pi}{4}, \frac{3 \pi}{4},-\frac{3 \pi}{4},-\frac{\pi}{4}\right\}
$$

$\lambda:=\sqrt{2}$, and $\left\{a_{k}\right\} \in \prod_{k=0}^{\infty} \mathbf{R}_{+}$and $\left\{c_{k 2}\right\} \in \prod_{k=0}^{\infty} \mathbf{R}_{+}$ are arbitrarily given so as to satisfy (B2) for $c_{k 1} \equiv 0$ (note that $a_{\lfloor s / 3\rfloor}$ and $c_{\lfloor s / 3\rfloor 2}$ express $a_{k}$ and $c_{k 2}$ for $\left.k=\lfloor s / 3\rfloor\right)$. If (C1)-(C3) hold for the G-SPSA algorithm with the same $f, \varepsilon_{k+}$, $\varepsilon_{k-}, \hat{\mathbf{T}}$ as in Theorem 2, $\left\{a_{k}\right\}, c_{k 1} \equiv 0,\left\{c_{k 2}\right\}$, the Bernoullitrial based distribution $\left\{\Xi_{k}\right\}, x_{0}:=x(0)$, and $x^{*}$, then $K$ is a solution to Problem 1 for any $r>0$.

Proof: The unicycle in (2) can move forward and turn at the same position; that is, $P$ can be steered straightforwardly to any translational position. Thus, (P1) holds for $\Delta \tau:=h$ and $\left(r_{1}, r_{2}, r_{3}\right):=(1,0,0)$. Then $u[s]$ in $(31)$ is composed of the control inputs for forward move and for turn at the same position, which can be related to (23) as follows. From (2) and the definition of the point-to-point control input $v$, the combination of the first and second inputs in (31) corresponds to

$$
v\left(t, 2 h, 0 \rightarrow\left[\begin{array}{c}
-c_{\lfloor s / 3\rfloor 2} \lambda\left[\begin{array}{c}
\cos (\zeta[s]) \\
\sin (\zeta[s])
\end{array}\right] \\
\zeta[s]
\end{array}\right], 1,0,0\right) .
$$

On the other hand, since $R_{2}(-\zeta[s])[\cos (\zeta[s]) \sin (\zeta[s])]^{\top}=$ $\left[\begin{array}{ll}1 & 0\end{array}\right]^{\top} \quad$ and $\quad \lambda[\cos (\zeta[s]) \quad \sin (\zeta[s])]^{\top}=$ $\left(\lambda[\cos (\zeta[s]) \quad \sin (\zeta[s])]^{\top}\right)^{(-1)}$ (the latter is related to Lemma 1 and it holds only for $\zeta[s]$ on the set in (32)), the third input corresponds to (34), shown at the bottom of the page. We see from (33) and (34) that the controller $K$ satisfies (23) for

$$
\begin{aligned}
& v\left(t, h, 0 \rightarrow\left[\lambda\left(c_{\lfloor s / 3\rfloor 2}-a_{\lfloor s / 3\rfloor} \frac{y_{p r e}[s]-y[s]}{c_{\lfloor s / 3\rfloor}}\right)\left[\begin{array}{l}
1 \\
0
\end{array}\right]\right], 1,0,0\right) \\
& =v\left(t, h, 0 \rightarrow\left[\begin{array}{r}
\left.\left.R_{2}(-\zeta[s])\left(c_{\lfloor s / 3\rfloor 2} \lambda\left[\begin{array}{c}
\cos (\zeta[s]) \\
\sin (\zeta[s])
\end{array}\right]-a_{\lfloor s / 3\rfloor} \hat{d}\left(y[s-1], y[s], c_{\lfloor s / 3\rfloor 2}, \lambda\left[\begin{array}{c}
\cos (\zeta[s]) \\
\sin (\zeta[s])
\end{array}\right]\right)\right)\right], 1,0,0\right) \\
0
\end{array}\right.\right.
\end{aligned}
$$




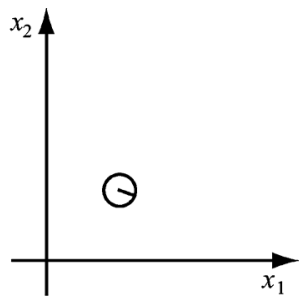

(a)

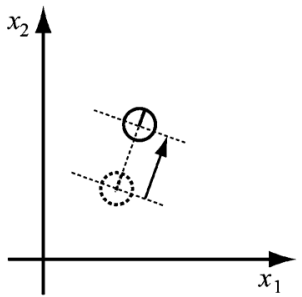

(c)

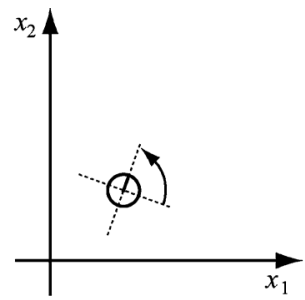

(b)

(d)

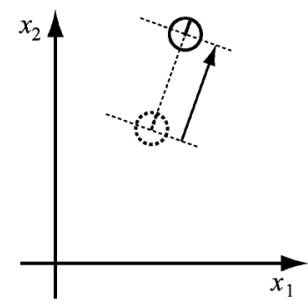

Fig. 4. Robot motion by the proposed controller in Theorem 3 (by repeating these steps, the robot is guided to the source). (a) Step 0: Current position. (b) Step 1: Random turn. (c) Step 2: Forward move. (d) Step 3: Forward or backward move.

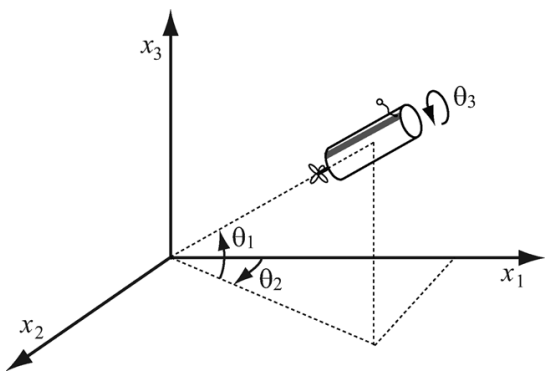

Fig. 5. 3-D nonholonomic mobile robot.

$\left\{t_{02}, t_{10}, t_{12}, t_{20}, \ldots\right\}:=\{2 h, 3 h, 5 h, 6 h, \ldots\},\left\{a_{k}\right\},\left\{c_{k 2}\right\}$, $\xi_{k}:=\lambda[\cos (\zeta[3 k]) \sin (\zeta[3 k])]^{\top}, \Delta \psi_{k 0}:=\zeta[3 k] \in \boldsymbol{\Phi}_{4}$, and $\Delta \psi_{k 2}:=0 \in \boldsymbol{\Phi}_{4}$. Here, (B2) holds for $\left\{a_{k}\right\},\left\{c_{k 2}\right\}$, and $c_{k 1} \equiv 0$. Furthermore, by noting that the probability distributions of $\zeta[s]$, the random vectors $\lambda[\cos (\zeta[3 k]) \sin (\zeta[3 k])]^{\top}$ $(k=0,1, \ldots)$ are equivalent to that from the elementwise Bernoulli trial shown in Section III-A, i.e., which satisfies (B1), (B3), and (B4). Therefore, this theorem follows from Theorem 1.

The proposed controller $K$ steers the robot $P$ as shown in Fig. 4. Three steps on a line segment are repeated: the random turn, the forward move, and the forward or backward move.

2) 3-D Nonholonomic Robot Case: In a similar way to the above, we can obtain a simple controller for a three-dimensional nonholonomic mobile robot.

Consider the robot in Fig. 5, which is described by

$$
P:\left[\begin{array}{c}
\dot{x}_{1}(t) \\
\dot{x}_{2}(t) \\
\dot{x}_{3}(t) \\
\dot{\theta}(t)
\end{array}\right]=\left[\begin{array}{cc}
\cos \theta_{1}(t) \cos \theta_{2}(t) & 0 \\
\cos \theta_{1}(t) \sin \theta_{2}(t) & 0 \\
\sin \theta_{1}(t) & 0 \\
0 & I_{3}
\end{array}\right] u(t)
$$

where $x(t):=\left[x_{1}(t) x_{2}(t) x_{3}(t)\right]^{\top} \in \mathbf{R}^{3}$ is the translational position, $\theta(t):=\left[\theta_{1}(t) \theta_{2}(t) \theta_{3}(t)\right]^{\top} \in \mathbf{R}^{3}$ is the orientational position (yaw, pitch, and roll), and $u(t) \in \mathbf{R}^{4}$ is the control input. This robot is the same as considered in [9].

For this, the following result is obtained.

Theorem 4: For the feedback system in Fig. 2, assume that $P$ is the three-dimensional robot in (35) and there exists a source $x^{*} \in \mathbf{R}^{3}$. Let $K$ be the modified version of (31) so that $u[s] \in$ $\mathbf{R}^{4}, \zeta[s] \in \mathbf{R}^{3}$ is the i.i.d. random vector drawn from the uniform distribution on

$$
\left\{-\tan ^{-1} \frac{1}{\sqrt{2}}, \tan ^{-1} \frac{1}{\sqrt{2}}\right\} \times\left\{\frac{\pi}{4}, \frac{3 \pi}{4},-\frac{3 \pi}{4},-\frac{\pi}{4}\right\} \times\{0\}
$$

the two 0 s for $s=1,4,7, \ldots$ and $s=2,5,8, \ldots$ are the threedimensional zero vectors, and $\lambda:=1 / \sin \left(\tan ^{-1}(1 / \sqrt{2})\right)$. If the same conditions in Theorem 3 hold, $K$ is a solution to Problem 1 for any $r>0$.

This can be derived in the same way to Theorem 3. Let $\zeta_{i}[s]$ denote the $i$ th element of $\zeta[s]$. Then, from the relation $\sin \left(\tan ^{-1}(1 / \sqrt{2})\right)=(1 / \sqrt{2}) \cos \left(\tan ^{-1}(1 / \sqrt{2})\right)$ and the probability distribution of $\zeta[s]$, it can be shown that the vectors

$$
\lambda\left[\begin{array}{c}
\cos \left(\zeta_{1}[s]\right) \cos \left(\zeta_{2}[s]\right) \\
\cos \left(\zeta_{1}[s]\right) \sin \left(\zeta_{2}[s]\right) \\
\sin \left(\zeta_{1}[s]\right)
\end{array}\right]
$$

$(s=0,3,6, \ldots)$, which correspond to $\xi_{k}$ (more precisely, $\left.\xi_{\lfloor s / 3\rfloor}\right)$ in (23), are equivalent to the random vectors from the elementwise Bernoulli trial. This is the main idea of the proof.

\section{Example}

Consider Problem 1, where the robot $P$ is the unicycle (2) and the signal field $S$ is given by the functions

$$
\begin{aligned}
f_{1}(x)= & \left(\left[\begin{array}{l}
x_{1} \\
x_{2}
\end{array}\right]-\left[\begin{array}{c}
110 \\
80
\end{array}\right]\right)^{\top}\left[\begin{array}{cc}
0.01 & -0.005 \\
-0.005 & 0.01
\end{array}\right] \\
& \times\left(\left[\begin{array}{l}
x_{1} \\
x_{2}
\end{array}\right]-\left[\begin{array}{c}
110 \\
80
\end{array}\right]\right) \\
f_{2}(x)= & \left(\left[\begin{array}{l}
x_{1} \\
x_{2}
\end{array}\right]-\left[\begin{array}{l}
90 \\
90
\end{array}\right]\right)^{\top}\left[\begin{array}{cc}
0.01 & 0.001 \\
0.001 & 0.003
\end{array}\right]\left(\left[\begin{array}{l}
x_{1} \\
x_{2}
\end{array}\right]-\left[\begin{array}{l}
90 \\
90
\end{array}\right]\right) \\
f_{3}(x)= & \left(\left[\begin{array}{l}
x_{1} \\
x_{2}
\end{array}\right]-\left[\begin{array}{l}
109 \\
110
\end{array}\right]\right)^{\top}\left[\begin{array}{cc}
0.02 & 0.003 \\
0.003 & 0.01
\end{array}\right]\left(\left[\begin{array}{l}
x_{1} \\
x_{2}
\end{array}\right]-\left[\begin{array}{l}
109 \\
110
\end{array}\right]\right)
\end{aligned}
$$

and the probability distribution $g(1)=0.15, g(2)=0.15$, and $g(3)=0.7$. Its source is

$$
\begin{aligned}
\operatorname{argmin}_{x \in \mathbf{R}^{2}} E\left[f_{\sigma}(x) \mid x\right] & =\operatorname{argmin}_{x \in \mathbf{R}^{2}} \sum_{\sigma=1}^{3} g(\sigma) f_{\sigma}(x) \\
& \simeq\left[\begin{array}{l}
109.13 \\
103.54
\end{array}\right] .
\end{aligned}
$$

The controller $K$ is given by Theorem 3 for $h:=1, a_{k}:=$ $15 /(k+1)^{0.55}$, and $c_{k 2}:=10 /(k+1)^{0.03}$.

Fig. 6 illustrates the contour plot of $E\left[f_{\sigma}(x) \mid x\right]$ and the moving trajectory of $P$ from the initial state $(x(0), \theta(0)):=$ $\left(\left[\begin{array}{ll}182 & 41\end{array}\right]^{\top}, 10\right)$, where the isosceles triangles express $\left(x\left(t_{k i}\right), \theta\left(t_{k i}\right)\right)(k=0,1, \ldots, \infty, i=0,2)$. Fig. 7 depicts the time evolution of the distance to the source, i.e., 


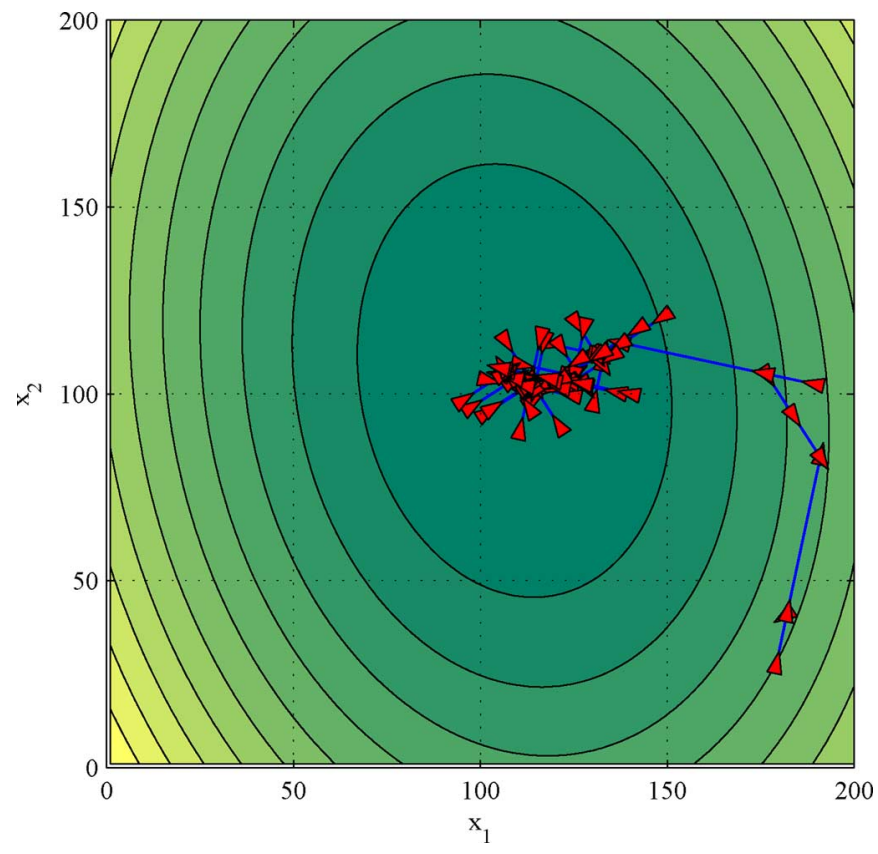

Fig. 6. Moving trajectory of unicycle by the proposed controller.

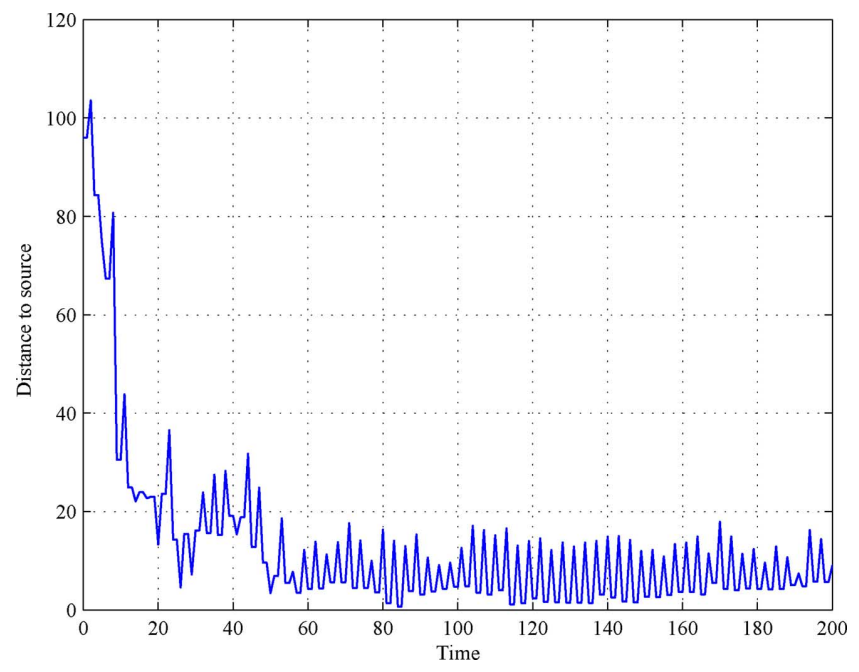

Fig. 7. Time evolution of the distance to the source.

$\left\|x(t)-\operatorname{argmin}_{x \in \mathbf{R}^{2}} E\left[f_{\sigma}(x) \mid x\right]\right\|$. We see that the robot $P$ is guided to the source by the simple controller.

Remark 6: As pointed out for the original SPSA algorithm [33], the choice of the gains $\left\{a_{k}\right\}$ and $\left\{c_{k 2}\right\}$ is critical to the performance, in particular, the convergence speed, of the G-SPSA based controllers. A practical way to determine these gains is the Monte Carlo method with randomly chosen $S$ based on some prior knowledge. There may be suitable selection for the stochastic source seeking, and such a topic is one of future works. Also, the best probabilistic distribution $\left\{\Xi_{k}\right\}$ should be clarified in the future, though the Bernoulli-type distribution has been mainly employed in this paper, by considering the optimality proven in [26] and the good property given by Lemma 1.

Remark 7: The above controllers are based on the turn and the forward/backward move. Even if the robot $P$ does not have such simple mobility, the source seeking can be achieved as long as (P1) holds.
Remark 8: Four limitations of the proposed method are noted. First, the signal fields $f_{\sigma}(x)$ have to be thrice differentiable as assumed in Section II-A. This guarantees that the expected value of $d\left(x_{k}, c_{k}, \xi_{k}, \delta_{k}\right)$ [in (10)] is nearly equal to the gradient of $f\left(x_{k}\right)$, from which the source seeking problem is solved. If this assumption does not hold (and the signal field $S$ does not switch), the method in [5] can be used as an alternative. Second, our source seeking controllers have been presented for periodic switching fields. The periodic switch is reasonable in some cases, e.g., in the base station placement, mentioned in Section I, with a large number of synchronized terminal units. Note, however, that the all results in this paper hold even for aperiodic fields which switch before discrete times $t_{k i}(k=0,1, \ldots, i=0,2)$ w.p.1. On the other hand, our result should be extended to more practical switches such as the Poisson switch. For the extention to the Poisson switch case, our result may be a basis because it is well-known that the Bimodal distribution, which is used as the switching model considered here, has a close relationship to the Poisson distribution [34]. Third, the proposed controllers cannot be applied to robots with nonzero constant velocity, such as aircraft. On the other hand, the methods in [4], [5], and [10] can be used for such robots. Finally, even when the robot can take the measurements continuously along the whole trajectory, the proposed controllers do not utilize the all of the data. If they are adopted for the gradient estimation, a better result could be obtained. It is however pointed out that the time for measurement is not always short, e.g., for chemical substances. Thus, one cannot always utilize the measurements along the whole trajectory.

Remark 9: We note the difference to other source seeking methods. First, a source seeking method for noisy environment has been developed in [13]. By using this result, a solution to Problem 1 may be obtained. The method, however, is based on the extremum seeking and can be applied to the only integrator and nonholonomic unicycles at present. On the other hand, the proposed method is given for robots satisfying (P1). Next, a method based on stochastic motion has been recently presented in [14]. Although it is similar to our method in the sense of taking stochastic motion, the method in [14] is a solution for non-switching fields and thus the performance for switching fields is unclear. Similarly to this, as mentioned in Section I, there are a number of source seeking methods and some of them might be useful for switching fields. However, there has been no theoretical result for switching fields.

\section{CONCLUSION}

A stochastic source seeking problem has been studied. By extending the SPSA algorithm in [21], we have developed source seeking controllers for randomly switching signal field. The key idea is to find a stochastic trajectory 1) converging to the unknown source with probability 1 and 2) followed by the robot without a position sensor. The trajectory is given by a generalized version of the SPSA algorithm. In addition, simple source seeking controllers have been provided for two types of nonholonomic robots, for which it has been shown that the source seeking is attained by the combination of simple movements.

As a first step for the switching source seeking, somewhat limited cases have been treated. In particular, the convergence 
of the proposed controllers is guaranteed for periodic and sufficiently fast switching fields. In the future, more general switching fields should be addressed. In addition, the proposed controllers cannot be used for robots with drift (i.e., movement under the zero control input), as stated in Remark 8. Solving this problem is left as a future work. Also, for various applications, the proposed framework should be extended to the case where the robot is disturbed by the environment (e.g., fluid environment) and to the stochastic source seeking by multiple robots.

\section{APPENDIX I}

\section{PROOF OF PROPOSITION 1}

\section{A. Notation}

We denote by $\mathrm{e}_{i}$ the $i$ th standard basis of $\mathbf{R}^{n}$. The Kronecker product of the vectors $y_{1}$ and $y_{2}$ is expressed as $y_{1} \otimes y_{2}$. For the row vector $z \in \mathbf{R}^{1 \times n}, \operatorname{diag}_{m}(z)$ expresses the block diagonal matrix whose $m$ diagonal blocks are $z$. Using this, we have the vector equality

$$
(z x) y=\operatorname{diag}_{m}(z)(y \otimes x)
$$

for $x \in \mathbf{R}^{n}, y \in \mathbf{R}^{m}$, and $z \in \mathbf{R}^{1 \times n}$. For the thrice differentiable function $f: \mathbf{R}^{n} \rightarrow \mathbf{R}$ and $j=2,3$, let

$$
\begin{aligned}
& f^{(1)}(x):=(\nabla f(x))^{\top}, \\
& f^{(j)}(x):=\left[\begin{array}{llll}
\frac{\partial f^{(j-1)}(x)}{\partial x_{1}} & \frac{\partial f^{(j-1)}(x)}{\partial x_{2}} & \cdots & \frac{\partial f^{(j-1)}(x)}{\partial x_{n}}
\end{array}\right] .
\end{aligned}
$$

Note that $f^{(j)}(x)$ is a row vector of dimension $n^{j}$. Using this, the function $f(x+y)$ (thrice differentiable) is expressed by Taylor's theorem as

$$
\begin{aligned}
f(x+y)=f(x)+f^{(1)}(x) y+ & \frac{1}{2} f^{(2)}(x)(y \otimes y) \\
& +\frac{1}{6} f^{(3)}(\bar{x})(y \otimes y \otimes y)
\end{aligned}
$$

where $\bar{x}$ is a vector on the line segment between $x$ and $x+y$.

\section{B. Proof}

By noting (A1), we apply Taylor's theorem in (37) to the terms $f\left(x_{k}+c_{k 1} T_{k} \xi_{k}\right)$ and $f\left(x_{k}-c_{k 2} T_{k} \xi_{k}\right)$ in (10), which gives

$$
\begin{aligned}
& f\left(x_{k}+c_{k 1} T_{k} \xi_{k}\right)-f\left(x_{k}-c_{k 2} T_{k} \xi_{k}\right) \\
& =\left(c_{k 1}+c_{k 2}\right) f^{(1)}\left(x_{k}\right) T_{k} \xi_{k} \\
& \quad+\frac{\left(c_{k 1}^{2}-c_{k 2}^{2}\right)}{2} f^{(2)}\left(x_{k}\right)\left(T_{k} \otimes T_{k}\right)\left(\xi_{k} \otimes \xi_{k}\right) \\
& \quad+\left(\frac{c_{k 1}^{3}}{6} f^{(3)}\left(\bar{x}_{k 1}\right)+\frac{c_{k 2}^{3}}{6} f^{(3)}\left(\bar{x}_{k 2}\right)\right)\left(T_{k} \otimes T_{k} \otimes T_{k}\right)\left(\xi_{k} \otimes \xi_{k} \otimes \xi_{k}\right)
\end{aligned}
$$

where $\bar{x}_{k 1}$ and $\bar{x}_{k 2}$ are vectors on the line segments between $x_{k}$ and $x_{k}+c_{k 1} T_{k} \xi_{k}$ and between $x_{k}$ and $x_{k}-c_{k 2} T_{k} \xi_{k}$, respectively. It follows from (10) and (36) that

$$
\begin{aligned}
& d\left(x_{k}, c_{k}, \xi_{k}, \delta_{k}\right) \\
& =T_{k}\left(f^{(1)}\left(x_{k}\right) T_{k} \xi_{k}\right) \xi_{k}^{(-1)}
\end{aligned}
$$

$$
\begin{aligned}
& +\frac{\left(c_{k 1}-c_{k 2}\right)}{2} T_{k}\left(f^{(2)}\left(x_{k}\right)\left(T_{k} \otimes T_{k}\right)\left(\xi_{k} \otimes \xi_{k}\right)\right) \xi_{k}^{(-1)} \\
& +\frac{1}{6} T_{k}\left(\left(\frac{c_{k 1}^{3}}{c_{k 1}+c_{k 2}} f^{(3)}\left(\bar{x}_{k 1}\right)+\frac{c_{k 2}^{3}}{c_{k 1}+c_{k 2}} f^{(3)}\left(\bar{x}_{k 2}\right)\right)\right. \\
& \left.\times\left(T_{k} \otimes T_{k} \otimes T_{k}\right)\left(\xi_{k} \otimes \xi_{k} \otimes \xi_{k}\right)\right) \xi_{k}^{(-1)} \\
& +T_{k} \frac{\varepsilon_{k+}-\varepsilon_{k-}}{\left(c_{k 1}+c_{k 2}\right)} \xi_{k}^{(-1)} \\
& =T_{k} \operatorname{diag}_{n}\left(f^{(1)}\left(x_{k}\right) T_{k}\right)\left(\xi_{k}^{(-1)} \otimes \xi_{k}\right) \\
& +\frac{\left(c_{k 1}-c_{k 2}\right)}{2} T_{k} \operatorname{diag}_{n}\left(f^{(2)}\left(x_{k}\right)\left(T_{k} \otimes T_{k}\right)\right) \\
& \times\left(\xi_{k}^{(-1)} \otimes\left(\xi_{k} \otimes \xi_{k}\right)\right) \\
& +\frac{1}{6} T_{k}\left(\left(\frac{c_{k 1}^{3}}{c_{k 1}+c_{k 2}} f^{(3)}\left(\bar{x}_{k 1}\right)+\frac{c_{k 2}^{3}}{c_{k 1}+c_{k 2}} f^{(3)}\left(\bar{x}_{k 2}\right)\right)\right. \\
& \left.\times\left(T_{k} \otimes T_{k} \otimes T_{k}\right)\left(\xi_{k} \otimes \xi_{k} \otimes \xi_{k}\right)\right) \xi_{k}^{(-1)} \\
& +T_{k} \frac{\varepsilon_{k+}-\varepsilon_{k-}}{\left(c_{k 1}+c_{k 2}\right)} \xi_{k}^{(-1)} .
\end{aligned}
$$

Here, from (B1), the relations

$$
E\left[\frac{\xi_{k j}}{\xi_{k i}} \mid x_{k}\right]=\left\{\begin{array}{ll}
0, & \text { if } i \neq j, \\
1, & \text { if } i=j,
\end{array} \quad E\left[\frac{\xi_{k j} \xi_{k l}}{\xi_{k i}} \mid x_{k}\right]=0\right.
$$

hold for every $(i, j, k, l) \in\{1,2, \ldots, n\}^{4}$ (note that (B1) a) implies $E\left[\xi_{k i}\right]=0$ and $\left.E\left[\xi_{k i}^{-1}\right]=0\right)$, from which we have

$$
\begin{aligned}
E\left[\xi_{k}^{(-1)} \otimes \xi_{k} \mid x_{k}\right] & =\left[\mathrm{e}_{1}^{\top} \mathrm{e}_{2}^{\top} \cdots \mathrm{e}_{n}^{\top}\right]^{\top} \\
E\left[\xi_{k}^{(-1)} \otimes\left(\xi_{k} \otimes \xi_{k}\right) \mid x_{k}\right] & =0_{n^{3} \times 1} .
\end{aligned}
$$

Moreover,

$$
\frac{c_{k i}^{3}}{c_{k 1}+c_{k 2}} \leq \frac{c_{k i}^{3}}{\max \left\{c_{k 1}, c_{k 2}\right\}} \leq c_{k i}^{2}=O\left(\left\|c_{k}\right\|^{2}\right) \quad(i=1,2)
$$

in (38). From (A2), (B1) (a), (38), (39), (40), and (41), we have

$$
\begin{aligned}
& E\left[d\left(x_{k}, c_{k}, \xi_{k}, \delta_{k}\right) \mid x_{k}\right] \\
& =T_{k} \operatorname{diag}_{n}\left(f^{(1)}\left(x_{k}\right) T_{k}\right) E\left[\xi_{k}^{(-1)} \otimes \xi_{k} \mid x_{k}\right]+O\left(\left\|c_{k}\right\|^{2}\right) \\
& =T_{k}\left[\begin{array}{c}
f^{(1)}\left(x_{k}\right) T_{k} \mathrm{e}_{1} \\
f^{(1)}\left(x_{k}\right) T_{k} \mathrm{e}_{2} \\
\vdots \\
f^{(1)}\left(x_{k}\right) T_{k} \mathrm{e}_{n}
\end{array}\right]+O\left(\left\|c_{k}\right\|^{2}\right) \\
& =T_{k}\left[\begin{array}{c}
\mathrm{e}_{1}^{\top} T_{k}^{\top} \nabla f\left(x_{k}\right) \\
\mathrm{e}_{2}^{\top} T_{k}^{\top} \nabla f\left(x_{k}\right) \\
\vdots \\
\mathrm{e}_{n}^{\top} T_{k}^{\top} \nabla f\left(x_{k}\right)
\end{array}\right]+O\left(\left\|c_{k}\right\|^{2}\right)
\end{aligned}
$$

as $c_{k} \rightarrow 0$. This, together with (A3), implies (12).

\section{APPENDIX II}

PROOF OF PROPOSITION 2

In a similar way to [21], Proposition 2 is proven via the Robbins-Monro Algorithm. 


\section{A. Robbins-Monro Algorithm}

The Robbins-Monro Algorithm has the form

$$
x_{k+1}=x_{k}-a_{k}\left(g\left(x_{k}\right)+b_{k}+e_{k}\right)
$$

where $x_{k} \in \mathbf{R}^{n}$ is the state, $a_{k} \in \mathbf{R}_{+}$is the gain, $g: \mathbf{R}^{n} \rightarrow \mathbf{R}^{n}$ is the function whose root is to be found, and $b_{k} \in(\mathbf{R} \cup\{\infty\})^{n}$ and $e_{k} \in \mathbf{R}^{n}$ are the random variables.

A result on the convergence is given by the following theorem (see, e.g., [25] for further details).

Proposition 3: Consider the algorithm in (42). Assume that there exists a root $x^{*} \in \mathbf{R}^{n}$ of the equation $g(x)=0$. If

(D1) $\lim _{k \rightarrow \infty} a_{k}=0$ and $\sum_{k=0}^{\infty} a_{k}=\infty$;

(D2) $\lim _{k \rightarrow \infty} b_{k}=0$ and $\sup _{k \in \mathbf{N}}\left\|b_{k}\right\|<\infty$ w.p.1;

(D3) for every $x_{0} \in \mathbf{S}$ and $\kappa \in \mathbf{R}_{+}$

$$
\lim _{k \rightarrow \infty} \operatorname{Prob}\left[\sup _{l \in[k, \infty)}\left\|\sum_{i=k}^{l} a_{i} e_{i}\right\| \geq \kappa\right]=0
$$

where $\mathbf{S}$ is a set defined in (D5);

(D4) $x^{*}$ is an asymptotically stable equilibrium of $\dot{x}(t)=$ $-g(x(t))$

(D5) there exists a compact stability region $\mathbf{S} \subseteq \mathbf{R}^{n}$ for $\dot{x}(t)=-g(x(t))$ (which is nonzero-measure and for which $\dot{x}(t)=-g(x(t))$ with $x(0)=x_{0}$ results in $\left.x(\infty)=x^{*}\right)$ such that $x_{k} \in \mathbf{S}$ infinitely often for every $x_{0} \in \mathbf{S}$ and almost all sample points;

(D6) $\sup _{k \in \mathbf{N}}\left\|x_{k}\right\|<\infty$ w.p.1 for every $x_{0} \in \mathbf{S}$; then

$$
\lim _{k \rightarrow \infty} x_{k}=x^{*} \text { w.p.1 }
$$

for every $x_{0} \in \mathbf{S}$.

\section{B. Proof of Proposition 2}

By Proposition 1 and the relation

$$
\begin{array}{r}
d\left(x_{k}, c_{k}, \xi_{k}, \delta_{k}\right)=E\left[d\left(x_{k}, c_{k}, \xi_{k}, \delta_{k}\right) \mid x_{k}\right]+d\left(x_{k}, c_{k}, \xi_{k}, \delta_{k}\right) \\
-E\left[d\left(x_{k}, c_{k}, \xi_{k}, \delta_{k}\right) \mid x_{k}\right]
\end{array}
$$

the G-SPSA algorithm given by (9) and (10) is represented as

$$
\begin{aligned}
x_{k+1}=x_{k}-a_{k} & \left(\nabla f\left(x_{k}\right)+O\left(\left\|c_{k}\right\|^{2}\right)\right. \\
+ & \left.d\left(x_{k}, c_{k}, \xi_{k}, \delta_{k}\right)-E\left[d\left(x_{k}, c_{k}, \xi_{k}, \delta_{k}\right) \mid x_{k}\right]\right)
\end{aligned}
$$

under (A1)-(A3) and (B1). Therefore, the G-SPSA algorithm with a fixed sequence $\left\{T_{k}\right\}$ is equivalent to (42) for

$$
\begin{aligned}
g\left(x_{k}\right) & =\nabla f\left(x_{k}\right) \\
b_{k} & =O\left(\left\|c_{k}\right\|^{2}\right) \\
e_{k} & =d\left(x_{k}, c_{k}, \xi_{k}, \delta_{k}\right)-E\left[d\left(x_{k}, c_{k}, \xi_{k}, \delta_{k}\right) \mid x_{k}\right] .
\end{aligned}
$$

Furthermore, under (A6), the statement

(*) for each sequence $\left\{T_{k}\right\} \in \prod_{k=0}^{\infty} \mathbf{T}, " \lim _{k \rightarrow \infty} x_{k}=x^{*}$ w.p.1" holds

implies the main statement in the theorem ("(13) holds for every $\left\{T_{k}\right\} \in \prod_{k=0}^{\infty} \mathbf{T}^{\prime \prime}$ holds w.p.1), since (A6) means that $\prod_{k=0}^{\infty} \mathbf{T}$ is a countable set.
So in the following part, to prove $(\star)$, we show under (45)-(47) that (A2)-(A5), (B2)-(B4), and (C1)-(C3) imply (D1)-(D6) for any $\left\{T_{k}\right\} \in \prod_{i=0}^{\infty} \mathbf{T}$.

First, it is trivial that (B2) implies (D1) and (D2) and that (A4), (C1), and (C2) imply (D4)-(D6) because $\mathbf{T} \subseteq \hat{\mathbf{T}}$.

Next, we prove that (D3) holds under (A2), (A3), (A5), (B2)-(B4), and (C3). Suppose that a sequence $\left\{T_{k}\right\} \in \prod_{k=0}^{\infty} \mathbf{T}$ is arbitrarily given and consider the stochastic process $\left\{\sum_{i=k}^{\beta} a_{i} e_{i}\right\}_{\beta=k}^{\infty}$ with the filtration $\mathcal{F}_{\beta}$ generated by $\left\{\left(x_{k}, \xi_{k}\right)\right\}_{i=0}^{\beta}$. From Jensen's inequality and the fact that $E\left[e_{i}^{\top} e_{j}\right]=0$ for $i \neq j$, we have

$$
\left(E\left[\left\|\sum_{i=k}^{\beta} a_{i} e_{i}\right\|\right]\right)^{2} \leq E\left[\left\|\sum_{i=k}^{\beta} a_{i} e_{i}\right\|^{2}\right]=\sum_{i=k}^{\beta} E\left[\left\|a_{i} e_{i}\right\|^{2}\right] .
$$

Furthermore, under (A3), (A5), (B2), (B4), and (C3), it can be shown in a similar way to [21] that

$$
\sum_{k=0}^{\infty} E\left[\left\|a_{k} e_{k}\right\|^{2}\right] \leq \mu \sum_{k=0}^{\infty} \frac{a_{k}^{2}}{\left\|c_{k}\right\|^{2}}<\infty
$$

where $\mu$ is a constant that does not depend on $k$. Equations (48) and (49) mean $E\left[\left\|\sum_{i=k}^{\beta} a_{i} e_{i}\right\|\right]<\infty$ for every $\beta \in$ $\{k, k+1, \ldots\}$. It follows that the sequences $\left\{\sum_{i=k}^{\beta} a_{i} e_{i}\right\}_{\beta=k}^{\infty}$ and $\left\{\left\|\sum_{i=k}^{\beta} a_{i} e_{i}\right\|\right\}_{\beta=k}^{\infty}$ are integrable. Thus, if the martingale difference assumption (A2) and the independence assumption (B3) hold, the sequence $\left\{\sum_{i=k}^{\beta} a_{i} e_{i}\right\}_{\beta=k}^{\infty}$ is martingale in the filtration $\mathcal{F}_{\beta}$ and further $\left\{\left\|\sum_{i=k}^{\beta} a_{i} e_{i}\right\|\right\}_{\beta=k}^{\infty}$ is submartingale in $\mathcal{F}_{\beta}$ (by the Jensen's inequality). This follows from the relation

$$
\begin{aligned}
& E\left[\sum_{i=k}^{\beta+1} a_{i} e_{i}-\sum_{i=k}^{\beta} a_{i} e_{i} \mid \sum_{i=k}^{\beta} a_{i} e_{i}\right] \\
& =a_{\beta+1} E\left[E\left[e_{\beta+1} \mid x_{0}, x_{1}, \ldots, x_{\beta}, \xi_{\beta}, \sum_{i=k}^{\beta} a_{i} e_{i}\right] \mid \sum_{i=k}^{\beta} a_{i} e_{i}\right] \\
& =a_{\beta+1} E\left[E\left[e_{\beta+1} \mid x_{0}, x_{1}, \ldots, x_{\beta}, \xi_{\beta}\right] \mid \sum_{i=k}^{\beta} a_{i} e_{i}\right]=0
\end{aligned}
$$

subject to (A2) and the fact that $\sum_{i=k}^{\beta} a_{i} e_{i}$ is determined by $\left\{\left(x_{k}, \xi_{k}\right)\right\}_{i=0}^{\beta}$. The submartingale property enables us to apply Doob's martingale inequality to the probability part of the left hand side of (43), which gives

$$
\operatorname{Prob}\left[\sup _{\beta \in[k, \infty)}\left\|\sum_{i=k}^{\beta} a_{i} e_{i}\right\| \geq \kappa\right] \leq \lim _{\beta \rightarrow \infty} \kappa^{-2} E\left[\left\|\sum_{i=k}^{\beta} a_{i} e_{i}\right\|^{2}\right] \text {. }
$$

Equations (48)-(50) imply

$$
\operatorname{Prob}\left[\sup _{\beta \in[k, \infty)}\left\|\sum_{i=k}^{\beta} a_{i} e_{i}\right\| \geq \kappa\right] \leq \kappa^{-2} \mu \sum_{i=k}^{\infty} \frac{a_{i}^{2}}{\left\|c_{i}\right\|^{2}} .
$$

This and (B2) prove (D3).

\section{ACKNOWLEDGMENT}

S.-i. Azuma would like to thank Prof. J. C. Spall, Johns Hopkins University, for his valuable information on one-sided 
SPSA algorithms. He also would like to thank Prof. T. Sugie, Kyoto University, for giving the opportunity to study at University of Pennsylvania.

\section{REFERENCES}

[1] A. A. Julius, M. S. Sakar, E. Steager, M. J. Kim, V. Kumar, and G. J. Pappas, "Harnessing bacterial power for micro scale manipulation and locomotion," in Proc. IEEE Int. Conf. Robot. Autom., 2009, pp. 1004-1009.

[2] S. D. Nathanson, "Insights into the mechanisms of lymph node metastasis," Cancer, vol. 98, no. 2, pp. 413-423, 2003.

[3] E. Burian, D. Yoerger, A. Bradley, and H. Singh, "Gradient search with autonomous underwater vehicles using scalar measurements," in Proc. IEEE Symp. Autonom. Underwater Veh. Technol., 1996, pp. 86-98.

[4] C. G. Mayhew, R. G. Sanfelice, and A. R. Teel, "Robust source-seeking hybrid controllers for nonholonomic vehicles," in Proc. Amer. Control Conf., 2007, pp. 1185-1190.

[5] C. G. Mayhew, R. G. Sanfelice, and A. R. Teel, "Robust hybrid source-seeking algorithms based on directional derivatives and their approximations," in Proc. 47th IEEE Conf. Decision Control, 2008, pp. $1735-1740$.

[6] A. R. Mesquita, J. P. Hespanha, and K. Åström, "Optimotaxis: A stochastic multi-agent optimization procedure with point measurements," in Hybrid Systems: Computation and Control, M. Egerstedt and B. Mishra, Eds. Berlin, Germany: Springer-Verlag, 2008, vol. 2623, Lecture Notes in Computer Science, pp. 358-371.

[7] K. B. Ariyur and M. Krstic, Real-Time Optimization by Extremum Seeking Feedback. New York: Wiley-Interscience, 2003.

[8] C. Zhang, D. Arnold, N. Ghods, A. Siranosian, and M. Krstic, "Source seeking with non-holonomic unicycle without position measurement and with tuning of forward velocity," Syst. Control Lett., vol. 56, no. 3, pp. 245-252, 2007.

[9] J. Cochran, A. Siranosian, N. Ghods, and M. Krstic, "3-D source seeking for underactuated vehicles without position measurement," IEEE Trans. Robot., vol. 25, no. 1, pp. 117-129, 2009.

[10] J. Cochran and M. Krstic, "Nonholonomic source seeking with tuning of angular velocity," IEEE Trans. Autom. Control, vol. 54, no. 4, pp. 717-731, Apr. 2009.

[11] J. Cochran, S. D. Kelly, X. Hailong, and M. Krstic, "Source seeking for a Joukowski foil model of fish locomotion," in Proc. Amer. Control Conf., 2009, pp. 1788-1793.

[12] J. Cochran, E. Kanso, and M. Krstic, "Source seeking for a three-link model of fish locomotion," in Proc. Amer. Control Conf., 2009, pp. $1808-1813$.

[13] M. S. Stankovic and D. M. Stipanovic, "Discrete time extremum seeking by autonomous vehicles in a stochastic environment," in Proc. Joint 48th IEEE Conf. Decision Control and 28th Chinese Control Conf., 2009, pp. 4541-4546.

[14] S. J. Liu and M. Krstic, "Stochastic source seeking for nonholonomic unicycle," Automatica, vol. 46, no. 9, pp. 1443-1453, 2010.

[15] B. Porat and A. Nehorai, "Localizing vapor-emitting sources by moving sensors," IEEE Trans. Signal Process., vol. 44, no. 4, pp. 1018-1021, Apr. 1996.

[16] P. Ogren, E. Fiorelli, and N. E. Leonard, "Cooperative control of mobile sensor networks: Adaptive gradient climbing in a distributed environment," IEEE Trans. Autom. Control, vol. 49, no. 8, pp. 1292-1302, Aug. 2004.

[17] S. Pang and J. A. Farrell, "Chemical plume source localization," IEEE Trans. Syst., Man, Cybern., B: Cybern., vol. 36, no. 5, pp. 1068-1080, Oct. 2006

[18] A. Teel and D. Popović, "Solving smooth and nonsmooth extremum seeking problems by methods of nonlinear programming," in Proc. Amer. Control Conf., 2001, pp. 2394-2399.

[19] C. Manzie and M. Krstic, "Extremum seeking with stochastic perturbations," IEEE Trans. Autom. Control, vol. 54, no. 3, pp. 580-585, Mar. 2009.

[20] S. Liu and M. Krstic, "Stochastic averaging in continuous time and its applications to extremum seeking," IEEE Trans. Autom. Control, vol. 55 , no. 10 , pp. $2235-2250$, Oct. 2010.

[21] J. C. Spall, "Multivariate stochastic approximation using a simultaneous perturbation gradient approximation," IEEE Trans. Autom. Control, vol. 37, no. 3, pp. 332-341, Mar. 1992.

[22] S. Azuma, M. S. Sakar, and G. J. Pappas, "Nonholonomic source seeking in switching random fields," in Proc. 49th IEEE Conf. Decision Control, 2010, pp. 6337-6342.

[23] R. M. Murray, Z. Li, and S. S. Sastry, A Mathematical Introduction to Robotic Manipulation. Boca Raton, FL: CRC, 1994.

[24] H. Nijmeijer and A. J. van der Schaft, Nonlinear Dynamical Control Systems. New York: Springer-Verlag, 1990.

[25] H. J. Kushner and D. S. Clark, Stochastic Approximation Methods for Constrained and Unconstrained Systems. New York: SpringerVerlag, 1978.
[26] P. Sadegh and J. C. Spall, "Optimal random perturbations for stochastic approximation using a simultaneous perturbation gradient approximation," in Proc. Amer. Control Conf., 1997, pp. 3582-3586.

[27] J. C. Spall, Introduction to Stochastic Search and Optimization. Hoboken, NJ: Wiley-Interscience, 2003.

[28] J. C. Spall, Simultaneous Perturbation Stochastic Approximation [Online]. Available: http://www.jhuapl.edu/SPSA/

[29] L. Ljung, "Strong convergence of a stochastic approximation algorithm," Annals of Statistics, vol. 6, no. 3, pp. 680-696, 1978.

[30] V. S. Borkar, Stochastic Approximation: A Dynamical Systems Viewpoint. Cambridge: Cambridge University Press, 2008.

[31] J. C. Spall, "A one-measurement form of simultaneous perturbation stochastic approximation," Automatica, vol. 33, no. 1, pp. 109-112, 1997.

[32] H. C. Berg and D. A. Brown, "Chemotaxis in Escherichia coli analysed by three-dimensional tracking," Nature, vol. 239, no. 5374, pp. 500-504, 1972.

[33] J. C. Spall, "Stochastic optimization," in Handbook of Computational Statistics: Concepts and Methods, J. E. Gentle, W. K. Haerdle, and Y. Mori, Eds. Berlin, Germany: Springer-Verlag, 2004, pp. 169-197.

[34] E. J. Borowski and J. M. Borwein, Collins dictionary of Mathematics, Second ed. London, U.K.: Collins, 2005, Collins Dictionary of Mathematics.

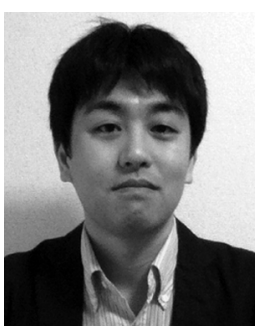

Shun-ichi Azuma (S'03-M'05) was born in Tokyo, Japan, in 1976. He received the B.Eng. degree in electrical engineering from Hiroshima University, Hiroshima, Japan, in 1999 and the M.Eng. and $\mathrm{Ph} . \mathrm{D}$. degrees in control engineering from the Tokyo Institute of Technology, Tokyo, Japan, in 2001 and 2004, respectively.

He was a Research Fellow of the Japan Society for the Promotion of Science at Tokyo Institute of Technology from 2004 to 2005 and an Assistant Professor in the Department of Systems Science, Graduate School of Informatics, Kyoto University, Uji, Japan, from 2005 to 2011. $\mathrm{He}$ is currently an Associate Professor at Kyoto University. He held visiting positions at the Georgia Institute of Technology, Atlanta, from 2004 to 2005 and at University of Pennsylvania, Philadelphia, from 2009 to 2010.

$\mathrm{He}$ has served as an Associate Editor of the IEEE CSS Conference Editorial Board since 2011. His research interests include analysis and control of hybrid systems.

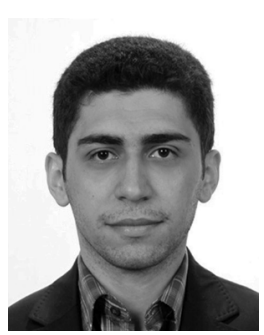

Mahmut Selman Sakar (M'10) received the Ph.D. degree in electrical and systems engineering from the University of Pennsylvania, Philadelphia, in 2010.

$\mathrm{He}$ is currently a Postdoctoral Associate in the Department of Mechanical Engineering, Massachusetts Institute of Technology, Cambridge. His research interests include single cell manipulation, microrobotics, bioengineered skeletal muscle microtissue, optogenetics, and foraging behavior of ant colonies.

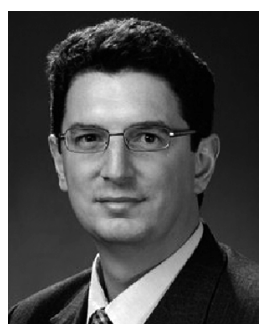

George J. Pappas (S'90-M'91-SM'04-F'09) received the Ph.D. degree in electrical engineering and computer sciences from the University of California, Berkeley, in 1998.

$\mathrm{He}$ is currently the Joseph Moore Professor of Electrical and Systems Engineering at the University of Pennsylvania, Philadelphia. He is a member of the General Robotics, Automation, Sensing, and Perception (GRASP) Laboratory and serves as the Deputy Dean for Research in the School of Engineering and Applied Science. His current research interests include hybrid and embedded systems, hierarchical control systems, distributed control systems, nonlinear control systems, with applications to robotics, unmanned aerial vehicles, biomolecular networks, and green buildings.

Dr. Pappas has received numerous awards, including the National Science Foundation (NSF) CAREER Award in 2002, the NSF Presidential Early Career Award for Scientists and Engineers in 2002, the 2009 George S. Axelby Outstanding Paper Award, the 2010 Anotnio Ruberti Outstanding Young Researcher Prize, and the Eliahu Jury Award for Excellence in Systems Research from the University of California, Berkeley. 